\title{
INVARIANT IMBEDDING AND HYPERBOLIC HEAT WAVES
}

\author{
DAVID J.N. WALL \\ Department of Mathematics, University of Canterbury \\ Private Bag 4800, Christchurch, New Zealand \\ and \\ PETER OLSSON \\ Division of Mechanics, Chalmers University of Technology \\ S-41296 Gothenburg, Sweden
}

No. 137

February, 1996

\begin{abstract}
This paper builds up a general wave splitting and imbedding theory for solution of both direct and inverse problems associated with thermal processes. It is done by using a full representation of the thermal phenomenon by virtue of Cattaneo's law. This law by ensuring finite thermal propagation speeds, enables an imbedding equation to layer strip the medium; so allowing the solution to the inverse problem of determination of a spatially varying diffusivity. Theoretical results and numerical algorithms are developed and numerical experiments are used to illustrate the effectiveness of the latter.
\end{abstract}




\title{
INVARIANT IMBEDDING AND HYPERBOLIC HEAT WAVES
}

\author{
DAVID J.N. WALL AND PETER OLSSON
}

\begin{abstract}
This paper builds up a general wave splitting and imbedding theory for solution of both direct and inverse problems associated with thermal processes. It is done by using a full representation of the thermal phenomenon by virtue of Cattaneo's law. This law by ensuring finite thermal propagation speeds, enables an imbedding equation to layer strip the medium; so allowing the solution to the inverse problem of determination of a spatially varying diffusivity. Theoretical results and numerical algorithms are developed and numerical experiments are used to illustrate the effectiveness of the latter.
\end{abstract}

\section{INTRODUCTION}

It is usually considered that the heat conduction in a thermally conducting solid is governed by the Fourier law, but then the resultant equation governing the dynamics of the heat flow is a parabolic equation and consequently has the unphysical property that the information propagates at infinite speed. Cattaneo [3] resolved this unphysical attribute by replacing Fourier's law by the more general one, since named after him, which we shall utilise in the sequel.

Wave splitting and invariant imbedding techniques have been very successful in their application to many inverse problems for hyperbolic equations. They have also been successful when applied to elliptic problems [14], but they have not been effective for parabolic equations. It has been shown by Vogel [17] layer stripping techniques are not suitable for parabolic equations. This paper is the outcome of our work towards the application of wave splitting and invariant imbedding techniques to phenomena, that are generally considered parabolic in nature. We concentrate, in the sequel, our ideas towards the evaluation of heat processes in solids through wave splitting techniques when Cattaneo's law is utilised. The literature in heat waves has grown considerably since Cattaneo, and an excellent review of the subject can be found in the two papers by Joseph and Preziosi [6],[7].

At room temperatures the the relaxation time $\tau$ is of the order $10^{-13}$ seconds and as the diffusivity in metallic conductors is of the order $10^{-5} \mathrm{~m}^{2} / \mathrm{s}$ this implies that the wave speed is of the order of the speed of sound $0.5 \times 10^{4} \mathrm{~m} / \mathrm{s}$. Therefore technology to resolve the inverse problem over dimensions of the order $10^{-9} \mathrm{~m}^{1}$ would therefore require femto-second laser technology. This technology is currently available. Hyperbolic heat waves are more

Date: February 1996.

1995 Physics and Astronomy Classification Scheme. PACS: 03.40Kf, 44.10, 44.30.

${ }^{1}$ For reasons made apparent by equation (36) inverse problems can only be resolved over dimensions of the order of the e-fold distance (defined after the aforementioned equation), which is of the order of $10^{-9} \mathrm{~m}$ for metallic conducors at room temperature. 
readily observed in super-cooled materials, such as liquid helium II; this could mean that appropriate inverse problems can be solved, so yielding further insight into such problems.

Perhaps the most meaningful application of our techniques, are to the solution of parabolic inverse problems when the wave speed of an associated hyperbolic problem is considered as a regularisation parameter. This approach will be considered in a later paper.

In Section 2 the prerequisite equations are developed. The wave splitting concept and some of its properties for appropriate second order equations is examined in Section 3 and this is extended to first order system equations in Section 4. In Section 5 the wave splitting is used to transform the equations of Section 2. The equations for the Green propagators are derived in Section 6 and this is followed by the equations derived by imbedding through the reflection kernel in Section 7. The discretisation of the equations derived in the earlier sections is discussed in Section 8 and some numerical examples of solutions for both the direct and the inverse problems are given in Section 9 .

\section{Preliminaries}

When heat waves are important the equation connecting the heat flux $q$, directed in the $x$-coordinate direction, to the temperature $T$ must at least have an extra thermal inertia term added, when compared to Fourier's conduction law. The Cattaneo equation for one-dimensional heat flow in a heat conducting solid has such a term and can be written as

$$
\tau \frac{\partial q}{\partial t}+q=-k \frac{\partial T}{\partial x}
$$

where $\tau$ is a relaxation time and $k$ is the thermal conductivity of the media. The relaxation time depends on the mechanism of heat transport, and represents the time lag needed to establish steady-state heat conduction in an element of volume when a temperature gradient is suddenly applied to that element. The other equation necessary to link temperature to the conduction heat flux, any lateral loss heat flux, $q_{\ell}$, and the internal rate of production of energy, $r$, is the conservation of internal energy equation

$$
\frac{\partial\left(c_{v} \rho T\right)}{\partial t}+\frac{\partial q}{\partial x}+\chi T=r
$$

Here $c_{v}$ is the specific heat at constant volume and $\rho$ is the mass density of the media. The lateral heat loss is assumed to be proportional to the temperature and is given by the term $\chi T^{2}$. These linear equations can be written as the system

$$
\partial_{x}\left[\begin{array}{l}
T \\
q
\end{array}\right]=\left[\begin{array}{cc}
0 & -\frac{\tau}{k} \partial_{t}-\frac{1}{k} \\
-\partial_{t} c_{v} \rho-\chi & 0
\end{array}\right]\left[\begin{array}{l}
T \\
q
\end{array}\right]+\left[\begin{array}{l}
0 \\
r
\end{array}\right],
$$

where in what follows, unless stated to the contrary, all coefficients in the partial differential equation will be assumed to be independent of the dependent variables but functions of the spatial variable $x$. The coefficients will further be assumed to be time independent;

\footnotetext{
${ }^{2} \mathrm{By}$ including this term, which is $\partial_{y} q_{\ell}=\chi T$, where $q_{\ell}$ is the heat flux in the lateral direction, say $y$, the problem is no longer one-dimensional. Of course no heat problem can be truly one dimensional, however if $\chi<<1$ the problem can be approximately treated as if it were.
} 
such an assumption holds for many materials (see [1] for an approach necessary for time dependent parameters). Throughout this paper it is assumed that the material parameters $c_{v}, \rho, k$, and $\tau$ are continuously differentiable and that $\chi$ is continuous in the region of interest.

We note that this system cannot be written as a second order partial differential equation; it is however possible to write it as such in two special cases, they are:

(i) $\tau \equiv$ constant - for the case where the dependent variable is the temperature

(ii) $\chi \equiv 0-$ for the case where the dependent variable is the heat flux.

These cases are now considered. If $\tau$ is dependent upon $x$ then it is possible to reduce the system into the following functional partial differential equation in $T$ - this is shown in Appendix A.

$$
\begin{aligned}
& \kappa^{-2} \tau \partial_{t}^{2} T+\kappa^{-2} \partial_{t} T-\partial_{x}^{2} T+\bar{a}(x) \partial_{x} T+\partial_{x}\left(\tau^{-1}\right) \rrbracket \\
& \quad
\end{aligned}
$$

where

$$
\bar{a}(x)=\partial_{x} \ln \left(\frac{\tau}{k}\right), \quad b(x)=\frac{\chi}{k},
$$

and the convolution integral operator

$$
\mathrm{J} f=\int_{0}^{t} J(t-s) f(s) d s, \quad \text { and } \quad J(t)=e^{-t / \tau} .
$$

It is now seen that this functional equation reduces to a partial differential equation when $\tau \equiv$ constant, so verifying item (i). We shall assume this is the case in the sequel, as the more general case will be more easily analysed through the system (2).

The parameters which are essential to our discussion in this paper are the diffusivity $\kappa^{2}=k /\left(c_{v} \rho\right),{ }^{3}$ the relaxation time $\tau$, and the thermal wave speed $c$, with $c^{-2}=\kappa^{-2} \tau$.

The other case occurs when $q$ is considered as the dependent variable, and when $\chi \equiv 0$, then the system (2) can be reduced to

$$
\kappa^{-2} \tau \partial_{t}^{2} q+\kappa^{-2} \partial_{t} q-\partial_{x}^{2} q+\bar{a}(x) \partial_{x} q=\bar{a}(x) r-\partial_{x} r
$$

where now

$$
\bar{a}(x)=\partial_{x} \ln \left(c_{v} \rho\right), \quad b \equiv 0 .
$$

When $\chi \neq 0$ the system (2) can be only reduced to a third order partial differential equation, which could also be handled by the techniques used in the sequel, however it is then preferable to use one of the other forms of equations we consider.

The equations (2), (3), and (5) with $r \equiv 0$, can now all be written in the system form as

$$
\partial_{x} \mathbf{u}=\mathbf{C u}+\mathbf{B u}
$$

\footnotetext{
${ }^{3}$ For convenience it is expedient for us to define diffusivity, $\kappa$ as the square of the usual terminology.
} 


\begin{tabular}{|l|l|}
\hline Heat Processes & Mass Transport \\
\hline$T$ - temperature & $c$ - mass concentration per unit volume \\
$q$ - heat flux & $q$ - mass flux \\
$c_{v}$ - specific heat at constant volume & 1 \\
$\rho$ - mass density & $\rho$ - mass density \\
$\kappa^{2}=\left(c_{v} \rho\right) / k$ - thermal diffusivity & $D^{2}-$ mass diffusivity \\
$k$ - thermal conductivity & $\rho / D^{2}$ \\
\hline
\end{tabular}

TABLE 1. The correspondence between variables for heat and mass transport

where for the second order equations $u$ denotes the appropriate dependent variable and where $\mathbf{u}=\left[\begin{array}{ll}u & \partial_{x} u\end{array}\right]^{T}$, and the matrices are

$$
\mathbf{C}=\left[\begin{array}{cc}
0 & 1 \\
\kappa^{-2}\left(\tau \partial_{t}^{2}+\partial_{t}\right) & 0
\end{array}\right], \quad \mathbf{B}=\left[\begin{array}{cc}
0 & 0 \\
b(x)\left(1+\tau \partial_{t}\right) & \vec{a}(x)
\end{array}\right]
$$

In the system (2) case the vector is $\mathbf{u}=\left[\begin{array}{ll}T & q\end{array}\right]^{T}$ and the matrices

$$
\mathbf{C}=\left[\begin{array}{cc}
0 & -\frac{1}{k}\left(1+\tau \partial_{t}\right) \\
-c_{v} \rho \partial_{t} & 0
\end{array}\right], \quad \mathbf{B}=\left[\begin{array}{cc}
0 & 0 \\
-\chi & 0
\end{array}\right]
$$

We should note that similar systems of equations can be written for one-dimensional mass transport processes where Cattaneo's law corresponds to a generalised Fick's law for mass diffusion. All of the methods developed in this paper can be also applied to such processes, with the appropriate translation of the dependent variables and material parameters as shown in Table 1.

We shall examine the system in $\S 4$, but initial examination of the second order equations is profitable for our exposition.

In the next section our attention will be on the $\mathrm{C}$ matrix, where we will diagonalise this operator matrix; the $\mathbf{B}$ matrix contains only terms irrelevant to this. We note that the $\mathbf{C}$ matrix has an extra diffusive derivative term, in the element $C_{12}$, the term $-\frac{\tau}{k} \partial_{t}$, when compared to the standard wave splitting for one dimensional wave equations. We include this term in $\mathbf{C}$ because any physically realistic media involving heat conduction must involve diffusion.

One feature of this investigation, is that for the inverse problem we assume that the measurements are carried out in a diffusive medium - not a ideal non-diffusive medium. Inverse problems similar to this have been examined by [5], [19]. However our objective to include the highly dissipative case - that is the diffusive one - is somewhat different to theirs, and as such the wave splitting operators we chose have a different form. Note that when wave propagation is present, the term dissipative and diffusive are the same.

The equations of this paper, besides also modelling heat and mass transport through hyperbolic waves, also model electromagnetic wave propagation problems in regions with dissipation; modelling such phenomenon as

- wave propagation at a termination of very lossy transmission lines 
- radio wave propagation through very attenuating media

- microwave resistance heating

\section{Wave Splitting for Second Order Equations}

It is now required to transform the equation (6) into a more convenient set of dependent variables, we do this by the technique of wave splitting (see [4] for a collection of articles). This transformation is motivated by formally diagonalising the matrix $\mathrm{C}$. The diagonalisation of the matrix $\mathbf{C}$ is less straightforward than when the pure wave equation is involved; linear algebra may be invoked to motivate the transformation in that case. In order to use linear algebra in it is necessary to first utilise the Laplace transform so that the various operators can be interpreted as pseudo-differential operators. Our notation for the transform variables is seen from the definition of the Laplace transform

$$
\widehat{\mathbf{u}}(x, s)=\int_{0}^{\infty} e^{-s t} \mathbf{u}(x, t) d t
$$

Our objective is to diagonalise $\mathrm{C}$, so it suffices to at first just transform (6) with the assumption $\mathbf{B} \equiv \mathbf{0}$. On noting that all the initial conditions used in the sequel will be of the form

$$
\mathbf{u}(x, 0)=D_{2} \mathbf{u}(x, 0)=0,
$$

we obtain the transformed equation

$$
\partial_{x} \widehat{\mathbf{u}}(x, s)=\widehat{\mathrm{C}} \widehat{\mathbf{u}}=\left[\begin{array}{cc}
0 & 1 \\
\kappa^{-2}\left(\tau s^{2}+s\right) & 0
\end{array}\right] \widehat{\mathbf{u}} .
$$

It is assumed that $\mathbf{u}$ is exponentially bounded in the $t$-variable. The eigenvalues of this matrix are found as $\pm \widehat{\lambda}$ where

$$
\widehat{\lambda}(s)=\kappa^{-1} \sqrt{s(\tau s+1)}
$$

where this is the Laplace transform representation of the pseudo-differential operator square root

$$
\left.\lambda=\mathcal{L}_{t}{ }^{1 / 2} \text { where } \mathcal{L}_{t} \equiv \kappa^{-2} \partial_{t}\left(\tau \partial_{t}+1\right)\right) .
$$

Use of the matrix of eigenvectors of $\widehat{\mathrm{C}}$

$$
\widehat{\mathbf{P}}=\left[\begin{array}{cc}
1 & 1 \\
-\widehat{\lambda} & \widehat{\lambda}
\end{array}\right],
$$

together with its inverse

$$
\widehat{\mathbf{P}}^{-1}=\frac{1}{2}\left[\begin{array}{cc}
1 & -\widehat{\lambda}^{-1} \\
1 & \widehat{\lambda}^{-1}
\end{array}\right]
$$

will diagonalise $\widehat{\mathrm{C}}$ as

$$
\widehat{\mathbf{C}} \widehat{\mathbf{P}}=\widehat{\mathbf{P}} \widehat{\Lambda} \quad \text { or } \quad \widehat{\mathbf{P}}^{-1} \widehat{\mathbf{C}} \widehat{\mathbf{P}}=\widehat{\Lambda}
$$


where

$$
\widehat{\Lambda}=\left[\begin{array}{cc}
-\widehat{\lambda} & 0 \\
0 & \hat{\lambda}
\end{array}\right] \text {. }
$$

The matrices $\boldsymbol{\Lambda}, \mathbf{P}$ and $\mathbf{P}^{-1}$ are all pseudo-differential operators and it is necessary for us to find their representation in the time domain in order to proceed. We examine them next.

First we examine the behaviour of the pseudo-differential operator defined by the inverse Laplace transformation of $\hat{\lambda}^{-1}(s) \widehat{f}(s)$. On finding the inverse transformation of $\hat{\lambda}^{-1}(s)$ it is found this is given by the convolution operator $\kappa \mathbb{K}$ where

$$
(\mathbb{K} f)(t)=\int_{0}^{t} K\left(x, t-t^{\prime}\right) f\left(t^{\prime}\right) d t^{\prime}
$$

with

So formally the operator

$$
K(x, t)=\frac{1}{\sqrt{\tau}} \exp \left(\frac{-t}{2 \tau}\right) \mathrm{I}_{0}(t / 2 \tau)
$$

$$
\lambda^{-1}=\kappa \mathbb{K}=\mathcal{L}_{t}^{-1 / 2} \text { where } \mathcal{L}_{t}^{-1 / 2} \equiv \kappa\left(\tau \partial_{t}^{2}+\partial_{t}\right)^{-1 / 2}
$$

Note that $\tau^{-1 / 2}$ has been kept in the definition of $\mathbb{K}$ so that the effect of $\tau \rightarrow 0$ can easily be determined. Throughout we use the assumption $f(0)=0$ (compare (7)).

Integration of $\mathbb{K} \circ \mathcal{L}_{t} f$ shows

$$
\mathbb{K} \circ \mathcal{L}_{t} f=\kappa^{-1}\left(\tau^{1 / 2} \partial_{t}+\frac{1}{2 \sqrt{\tau}}(1-\mathbb{L})\right) f(t),
$$

where

$$
(\mathbb{L} f)(t)=\int_{0}^{t} L\left(x, t-t^{\prime}\right) f\left(t^{\prime}\right) d t^{\prime}
$$

and

$$
L(x, t)=\exp \left(\frac{-t}{2 \tau}\right) \frac{\mathrm{I}_{1}(t / 2 \tau)}{t} .
$$

Now use of Liebnitz's rule on $\mathcal{L}_{t} \circ \mathbb{K} f$ also yields the left-hand-side of (11), so proving the commutation relationship

$$
\mathcal{L}_{t} \circ \mathbb{K} f=\mathbb{K} \circ \mathcal{L}_{t} f
$$

which appears in many wave splitting problems; in fact derivative operators up to order two commute with $\mathbb{K}$, that is

$$
\partial_{t} \mathbb{K} f=\mathbb{K} \partial_{t} f, \quad \partial_{t}^{2} \mathbb{K} f=\mathbb{K} \partial_{t}^{2} f,
$$

provided also $f^{\prime}(0)=0$. Motivated by the the commutation of pseudo-differential operators under composition, we write for the square root operators

$$
\mathcal{L}_{t}{ }^{1 / 2}(f)=\mathcal{L}_{t}{ }^{-1 / 2} \circ \mathcal{L}_{t}(f)=\mathcal{L}_{t} \circ \mathcal{L}_{t}{ }^{-1 / 2}(f) .
$$


Now $\mathcal{L}_{t}{ }^{-1 / 2}=\kappa \mathbb{K}$, and also observe (10), so from (15) and (13) we have the identification $\mathcal{L}_{t}{ }^{1 / 2}=\kappa \mathbb{K} \mathcal{L}_{t}$ which also suggests that this is $\kappa^{-1} \mathbb{K}^{-1}$ hence it follows from (11) that

$$
\mathbb{K}^{-1} f=\left(\tau^{1 / 2} \partial_{t}+\frac{1}{2 \sqrt{\tau}}(1-\mathbb{L})\right) f(t) .
$$

To prove that this is $\mathbb{K}^{-1}$, it is straightforward to show the inverse relation

$$
\mathbb{K} \circ \mathbb{K}^{-1} f=f, \quad \mathbb{K}^{-1} \circ \mathbb{K} f=f,
$$

is verified from the identity (see [15], page 320, formula 13)

$$
\int_{0}^{x} \frac{\mathrm{I}_{1}(x-y)}{(x-y)} \mathrm{I}_{0}(y) d y=\mathrm{I}_{1}(x)
$$

Furthermore, using $\mathcal{L}_{t}{ }^{1 / 2}=\kappa \mathbb{K} \mathcal{L}_{t}$ and integration by parts it can be shown that

$$
\mathbb{I}^{-1} f=\partial_{t} \mathbb{K} \circ\left(\tau \partial_{t}+1\right) f
$$

or equivalently by using the commutation property (13). Looking at (13) one would expect $\mathcal{L}_{t}=\mathcal{L}_{t}{ }^{1 / 2} \circ \mathcal{L}_{t}{ }^{1 / 2}$ or

$$
\mathbb{K}^{-1} \circ \mathbb{K}^{-1}=\mathcal{L}_{t}
$$

and this can be proven from (11).

The form of the operator $\mathbb{K}^{-1}$ is critical in obtaining a form reducing to the appropriate limit as $\tau \rightarrow 0$; representation (17) is the appropriate form. It is interesting to note that the approach in $\S 4$ automatically produces the correct form for the limiting procedure. The $\mathbb{K}$ operator is smoothing, compact on $L_{2}$, and as such the inverse operator $\mathbb{K}^{-1}$ is unbounded and ill-posed on $L_{2}$, even though existence of the operator has been proven by construction.

Theorem 1. The operators are injective and into on the spaces

$$
\begin{gathered}
\mathbb{K}: H^{s} \mapsto H^{s+i+1 / 2} \\
\mathbb{K}^{-1}: H^{s} \mapsto H^{s-i-1 / 2}
\end{gathered}
$$

when $\tau=0, i=0$ and when $\tau>0, i=1 / 2$.

Proof. To prove the operators are injective it is only necessary to look at the image of the zero function because the operators are linear; it follows trivially from their explicit form they are injective. The mapping properties of the operators follows directly from their Laplace transforms and the symbol mapping theorem [16],( pages 49 et. seq. ).

It is necessary to look at the forms that the maps $\mathbf{P}$ and $\mathbf{P}^{\mathbf{- 1}}$ take as the parameters take various limits. Of major concern here, is the limiting forms of the operators $\lambda$ and $\lambda^{-1}$ as $\tau \rightarrow 0$; when $\tau=0$, the model equations are parabolic. Define

$$
\mathbb{H} f=\int_{0}^{t} H(t-s) f(s) d s
$$


where

$$
H(t)=\frac{1}{\sqrt{\pi t}}
$$

and $\mathbb{H} f$ is related to the half derivative of $f$, that is $\mathbb{H} f=\partial_{t}^{-1 / 2} f$ and with composition properties [13]

$$
\partial_{t}^{1 / 2} f=\partial_{t} \mathbb{H} f=\partial_{t} \partial_{t}^{-1 / 2} f
$$

It is then possible to show

$$
\lim _{\tau \rightarrow 0} \kappa \mathbb{K}=\kappa \mathbb{H},
$$

and when representation (17) is used for $\mathbb{K}^{-1}$

$$
\lim _{\tau \rightarrow 0} \kappa^{-1} \mathbb{K}^{-1}=\kappa^{-1} \partial_{t} \mathbb{H}
$$

Therefore in the limit of the hyperbolic equations becoming parabolic, the splitting operators reduce to Vogel's [17] results. However as noted in the Introduction layer stripping techniques are not suitable for the parabolic heat equation.

Now looking at the limit as $\kappa^{-1} \rightarrow 0$, while keeping $\kappa^{-1} \sqrt{\tau} \rightarrow c^{-1}$ fixed, then the equation becomes non-diffusive. Equivalent to this is to allow $\tau \rightarrow \infty$, while again keeping $c$ fixed. So that

$$
\lim _{\tau \rightarrow \infty} \kappa \mathbb{K}=c \partial_{t}^{-1}
$$

and if the representation (16) is taken for $\mathbb{K}^{-1}$ it is found that

$$
\lim _{\tau \rightarrow \infty} \kappa^{-1} \mathbb{K}^{-1}=c^{-1} \partial_{t}
$$

These are the standard splittings for the wave equation.

We collect the matrices, in the time domain, central to the later development

$$
\mathbf{P}=\left[\begin{array}{cc}
1 & 1 \\
-\kappa^{-1} \mathbb{K}^{-1} & \kappa^{-1} \mathbb{K}^{-1}
\end{array}\right], \quad \mathbf{P}^{-1}=\frac{1}{2}\left[\begin{array}{cc}
1 & -\kappa \mathbb{K} \\
1 & \kappa \mathbb{K}
\end{array}\right] .
$$

\section{Wave Splitting for System}

On Laplace transforming the system (6), when $\mathbf{C}$ is appropriate for equation (2), the $\widehat{\mathbf{C}}$ matrix is then found to be

$$
\widehat{\mathbf{C}}=\left[\begin{array}{cc}
0 & -\frac{\tau}{k} s-\frac{1}{k} \\
-c_{v} \rho s & 0
\end{array}\right]
$$

and the eigenvalues of this matrix are identical to those for the $\widehat{\mathrm{C}}$ matrix of $\S 3$, namely (9), except now the matrices of eigenvectors are

$$
\widehat{\mathbf{P}}=\left[\begin{array}{cc}
1 & 1 \\
k \widehat{\lambda}(\tau s+1)^{-1} & -k \widehat{\lambda}(\tau s+1)^{-1}
\end{array}\right], \quad \widehat{\mathbf{P}}^{-1}=\frac{1}{2}\left[\begin{array}{cc}
1 & \frac{1}{k}(\tau s+1) \hat{\lambda}^{-1} \\
1 & -\frac{1}{k}(\tau s+1) \hat{\lambda}^{-1}
\end{array}\right] .
$$


The operators in these matrices need to be identified in terms of those from the last section, some manipulations of the transform equations then show

$$
\mathbf{P}=\left[\begin{array}{cc}
1 & 1 \\
k \kappa^{-1} \tilde{\mathbb{K}}^{-1} & -k \kappa^{-1} \widetilde{\mathbb{K}}^{-1}
\end{array}\right], \quad \mathbf{P}^{-1}=\frac{1}{2}\left[\begin{array}{cc}
1 & k^{-1} \kappa \widetilde{\mathbb{K}} \\
1 & -k^{-1} \kappa \widetilde{\mathbb{K}}
\end{array}\right],
$$

where $\widetilde{\mathbb{K}}^{-1}=\mathbb{K} \partial_{t}$ and $\widetilde{\mathbb{K}}=\mathbb{K} \circ\left(\tau \partial_{t}+1\right)$. It is straightforward to show that $\widetilde{\mathbb{K}}$ has the inverse $\widetilde{\mathbb{K}}^{-1}$.

In $\S 5$ the matrix $\mathbf{P}$ will be used to transform the problem through a linear transformation like

$$
\mathbf{u}=\mathbf{P v}
$$

where $\mathbf{v}$ is the new dependent variable. Examination of the dimensions of the component maps of $\mathbf{P}$ then will show the following results. The map $k \kappa^{-1} \tilde{\mathbb{K}}^{-1}$, at the plane $x=$ constant, maps the temperature field onto the heat flux, that is it provides a trace transformation - the Dirichlet-to-Neumann map. Note also the correspondence between these equations and the ones for the transmission line equations (see for example [2]), with the elements $k \kappa^{-1} \widetilde{\mathbb{K}}^{-1}$ having the dimensions of admittance $q / T$. These operators are not smoothing or differentiating, unless $\tau=0$, and as such are easier operators to perform numerical calculations with. This is typical behaviour for the system form of the equations.

Theorem 2. The operators are injective and into on the spaces

$$
\begin{gathered}
\widetilde{\mathbb{K}}: H^{s} \mapsto H^{s+i} \\
\widetilde{\mathbb{K}}^{-1}: H^{s} \mapsto H^{s-i}
\end{gathered}
$$

when $\tau=0, i=1 / 2$, and when $\tau>0, i=0$.

Proof. Injectivity follows from Theorem 1 and the mapping properties follows from the symbols of the operators as in Theorem 1.

It is important to note the diagonal matrix $\Lambda$ is the same as (21) so that the principal part of the dynamics equation will be the same for system (2) and the second order equations (3) and (5).

\section{System Dynamics}

Now on use of the diagonalising transformations

$$
\boldsymbol{v}^{ \pm}=\mathbf{P}^{-1} \mathbf{u}
$$

the equation (6) converts to

$$
\partial_{x} \boldsymbol{v}^{ \pm}=\mathcal{A} \boldsymbol{v}^{ \pm}
$$

with the new basis $v^{ \pm}=\left[\begin{array}{ll}v^{+} & v^{-}\end{array}\right]^{T}$, where $\left\{v^{+}, v^{-}\right\}$have the properties of right and left moving waves; we shall discuss this point further. 


\begin{tabular}{l|c|c}
\hline Coefficient & $\begin{array}{c}\text { Second order equations }(3)^{a} \\
\text { and }(5)^{b}\end{array}$ & ${\text { System }(2)^{c}}^{c}$ \\
\hline$d(x)$ & $-\frac{1}{2} \partial_{x}(\ln c)=-\frac{1}{2} \partial_{x}\left(\ln \left(\frac{\kappa}{\sqrt{\tau}}\right)\right)$ & $\frac{1}{2} \partial_{x}\left(\ln \left(\frac{k}{\kappa \sqrt{\tau}}\right)\right)=\frac{1}{2} \partial_{x}\left(\ln \left(\frac{k}{c \tau}\right)\right)$ \\
$e(x)$ & $-\frac{1}{4} \tau^{-1} \partial_{x}(\ln \tau)$ & $\frac{1}{4} \tau^{-1} \partial_{x}(\ln \tau)$ \\
$f(x)$ & $\frac{\kappa \chi}{2 k} \widetilde{\mathbb{K}}=\frac{\kappa \chi}{2 k} \mathbb{K} \circ\left(\tau \partial_{t}+1\right)$ & $\frac{\kappa \chi}{2 k} \widetilde{\mathbb{K}}$ \\
$g(x)$ & $\frac{1}{2} \partial_{x}\left(\ln \left(\frac{\tau}{k}\right)\right)$ for $(3)$ & 0 \\
& $\frac{1}{2} \partial_{x}\left(\ln \left(c_{v} \rho\right)\right)$ for $(5)$ & \\
\hline${ }^{a}$ Note in this case $\partial_{x} \ln \tau=0$ for coefficients $d, e$, and $g$. \\
${ }^{b}$ Note in this case $\chi=0$. \\
${ }^{c}$ Note the commutation property of $\mathrm{J}$ with $\partial_{t}$ has been used for coefficient $e$. \\
\hline
\end{tabular}

TABLE 2. Identification of parameters $d, e, f$, and $g$ for hyperbolic heat waves

$\left(\mathcal{A}-\partial_{x}+\partial_{t}\right)$ is the infinitesimal generator of the Banach space valued vector flow field, with

$$
\mathcal{A}=\Lambda+D
$$

$\Lambda$ being the diagonal operator matrix

$$
\Lambda=\left[\begin{array}{cc}
-\kappa^{-1} \mathbb{K}^{-1} & 0 \\
0 & \kappa^{-1} \mathbb{K}^{-1}
\end{array}\right]
$$

and where the dynamics matrix $D$ is

$$
D=-\mathbf{P}^{-1}\left(\partial_{\mathrm{x}} \mathbf{P}\right)+\mathbf{P}^{-1} \mathbf{B P} .
$$

For notational convenience we express the dynamics matrix as

$$
\boldsymbol{D}=\left[\begin{array}{ll}
\alpha & \beta \\
\gamma & \delta
\end{array}\right]
$$

Then in terms of the material parameters the system dynamics are

$$
\mathbf{P}^{-1}\left(\partial_{x} \mathbf{P}\right)=\left[\begin{array}{cc}
1 & -1 \\
-1 & 1
\end{array}\right]\left(d(x)+e(x) \partial_{t} \mathrm{~J}\right),
$$

with $\mathrm{J}$ is as in (4) and

$$
\mathbf{P}^{-1} \mathbf{B P}=f(x)\left[\begin{array}{cc}
-1 & -1 \\
1 & 1
\end{array}\right]+g(x)\left[\begin{array}{cc}
1 & -1 \\
-1 & 1
\end{array}\right]
$$

The coefficients in these equations for the various cases is shown in Table 2.

In the limiting case of parabolic heat flow, then $\tau \rightarrow 0$, the system dynamics is the same as in (20) but with the following replacements. The operator $\kappa^{-1} \mathbb{K}^{-1}$ in (21) is replaced 


\begin{tabular}{l|c|c}
\hline Coefficient & $\begin{array}{c}\text { Second order equations (3) } \\
\text { and }(5)^{a}\end{array}$ & System (2) \\
\hline$d(x)$ & $-\frac{1}{2} \partial_{x}(\ln \kappa)$ & $\frac{1}{2} \partial_{x}\left(\frac{k}{\kappa}\right)$ \\
$e(x)$ & 0 & 0 \\
$f(x)$ & $\frac{\kappa \chi}{2 k} \mathbb{H}$ & $\frac{\kappa \chi}{2 k} \mathbb{H}$ \\
$g(x)$ & $-\frac{1}{2} \partial_{x} \ln (k)$ for $(3)$ & 0 \\
$\frac{1}{2} \partial_{x} \ln \left(c_{v} \rho\right)$ for $(5)$ & \\
\hline Note in this case $\chi=0$.
\end{tabular}

TABLE 3. Identification of parameters $d, e, f$, and $g$ for parabolic heat conduction

by $\kappa^{-1} \partial_{t} \mathbb{H}$, and equations (23) and (24) hold except the material coefficients are given in Table 3 .

If $\mathbf{B} \equiv \mathbf{0}$ then $\mathcal{A}=\boldsymbol{\Lambda}$, and the system is decoupled into right and left moving thermal waves, respectively denoted by $v^{+}$and $v^{-}$. We now discuss the interpretation of the $v^{ \pm}$. For concreteness we just consider $v^{+}$. Examination of the system dynamics, when $\mathbf{B} \equiv 0$ and the remaining parameters are homogeneous, shows the right going wave must satisfy

$$
\left(\partial_{x}+\kappa^{-1} \mathbb{K}^{-1}\right) v^{+}=0
$$

In the special case $\tau \rightarrow \infty$, with $c$ fixed, this becomes

$$
\left(\partial_{x}+c^{-1} \partial_{t}\right) v^{+}=0
$$

which is satisfied by solutions of the form $v\left(c^{-1} x-t\right)$, the well known right going waves having Galilean translational invariance. When considering the more general operator found in (25), we cannot expect this to exhibit such symmetry because the wave will be attenuated as it moves to the right. However we still call right moving waves those that satisfy (25). From the splitting (18) it follows

$$
v^{+}=\frac{1}{2}\left(T+k^{-1} \kappa \widetilde{\mathbb{K}} q\right)
$$

for the equations derived from (2). Elementary calculations using the equations (2) and (16) shows (26) does indeed satisfy (25). Similarly it can be shown (25) is satisfied by the equations for the second order equations (3) and (5). Similar interpretations can be made for left-going waves.

When the material properties are not homogeneous we cannot make this physical interpretation for $v^{ \pm}$, however we shall still call such waves left and right moving waves for convenience. It should be apparent the mathematics still makes sense in that $\boldsymbol{v}^{ \pm}$satisfy (19). 


\section{The Direct Problem and the Green Operators}

The Green operators provide the mapping of the incident field at the boundary of a slab of the propagation medium to an interior point. These operators were first introduced by Krueger and Ochs [9], and because they satisfy linear functional equations have been found to provide efficient algorithms for solution of the direct problem. They also provide a method of solution which is different from the invariant imbedding method. This enables verification of the consistency of any numerical solutions to be obtained by comparing solutions obtained by the two methods. The thermal processes within the medium of the

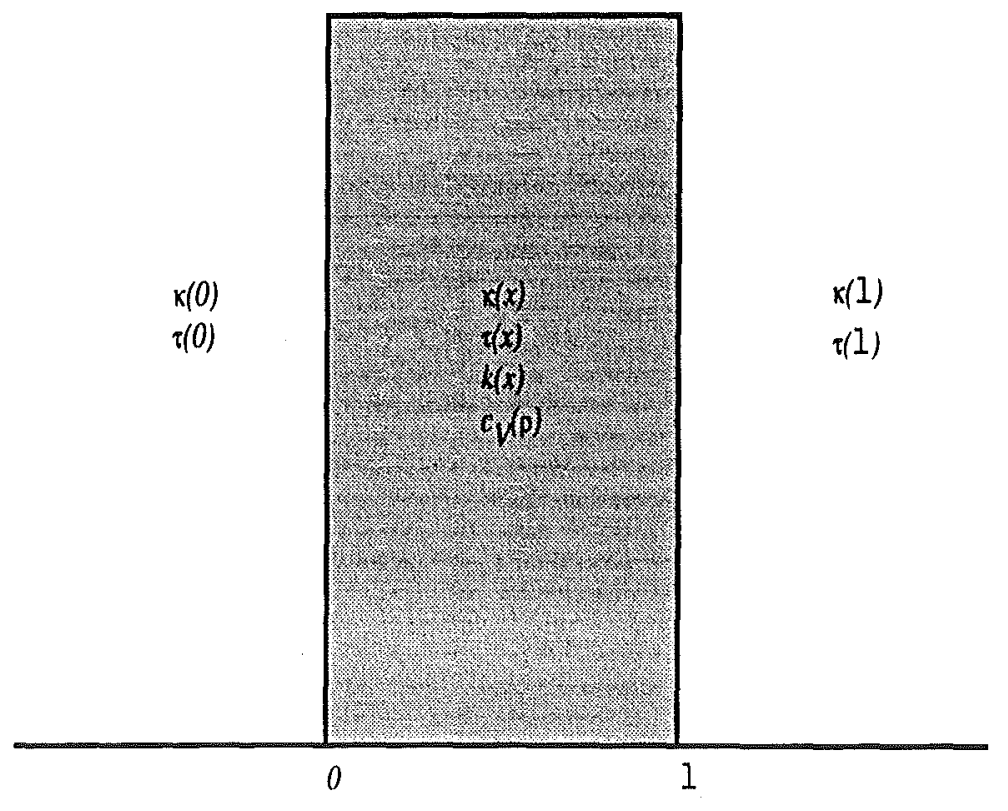

Figure 1. The slab geometry

slab $0<x<\ell$ are described by equations (6) and with a homogeneous medium outside this region the waves are also described by the same equations, but with a constant wave speed

$$
c(x)= \begin{cases}c(0) & x<0 \\ c(\ell) & x>\ell .\end{cases}
$$

This condition ensures that the thermal wave is matched (that is not reflected) at the boundaries of the slab $[0, \ell]$. If $c$ has a jump discontinuity it is still possible to treat the problem (see for example [8]), by our methods but we shall not consider such problems here. Note it is not necessary to make the assumption that $\kappa$ is continuous through the interfaces, but then it is required that

$$
\tau(x)= \begin{cases}\kappa(0) / c(0) & x<0 \\ \kappa(\ell) / c(\ell) & x>\ell .\end{cases}
$$

However in any realistic thermal conduction process, the attenuation will often be sufficient that for all intents the slab can be considered as semi-infinite. This is because the wave 
amplitude will be so small by the time the wave reaches the far slab boundary, little will remain to be reflected. With little loss of generality we take $\chi$ identically zero outside of $[0, \ell]$.

When considering the slab $x \in[0, \ell]$ we can define Green operators such that for $t>0$

$$
\begin{aligned}
& v^{-}(x, t+\zeta(x))=\mathrm{G}^{-} \circ v^{+}(0, t), \\
& v^{+}(x, t+\zeta(x))=a(x) v^{+}(0, t)+\mathrm{G}^{+} \circ v^{+}(0, t),
\end{aligned}
$$

where $a$ is the attenuation of a wave propagating from the interface $x=0$ to a point within the slab, $x$, and $\zeta(x)$ is the propagation time taken by a wave front to get there. Causality requires that $v^{ \pm}(x, t)=0$ for $t \leq \zeta(x)$. It is seen that the positive moving field at some point $x>0$ consists of two parts. The first part is due to the direct transmission of the incident field $v^{+}(0, t)$ with attenuation and time delay, and the second part is due to scattering effects in the slab - this is provided by $\mathrm{G}^{+} \circ v^{+}(0, t)$. The other Green operator provides the mapping between the incident right going wave $v^{+}(0, t)$ and a left going wave at $x>0$.

The Green operators can be shown to be convolutional operators of the form

$$
\left(\mathrm{G}^{ \pm} \circ f\right)(t)=\int_{0}^{t} G^{ \pm}(t-s) f(s) d s
$$

by the Duhamel integral principle. Insertion of (27)-(29) in (19) shows that the Green kernels satisfy the functional equations

$$
\begin{gathered}
\partial_{x} G^{+}=\frac{1}{2 \kappa \sqrt{\tau}}\left(a L+L * G^{+}-G^{+}\right)+\alpha G^{+}+\beta G^{-} \\
\partial_{x} G^{-}-2 c^{-1} \partial_{t} G^{-}=\frac{1}{2 \kappa \sqrt{\tau}}\left(G^{-}-L * G^{-}\right)+\delta G^{-}+\gamma G^{+},
\end{gathered}
$$

where $L * f$ denotes the time convolution operator with the kernel $L$ given by (12), namely

$$
L * f(x, t)=\int_{0}^{t} L\left(x, t-t^{\prime}\right) f\left(t^{\prime}\right) d t^{\prime} .
$$

The system (30) and (31) has boundary and initial conditions

$$
\begin{aligned}
G^{+}(0, t) & =0, \\
G^{+}(x, 0) & =\frac{a(x)}{2} \int_{0}^{x}\left(\frac{1}{4 \kappa \tau^{3 / 2}}-\gamma \beta c\right) d s \\
G^{-}(\ell, t) & =0, \\
G^{-}(x, 0)+\frac{1}{2} c \gamma a & =0,
\end{aligned}
$$

Here the multiplicative attenuation factor of the thermal wave propagating from $x=0$ to $x$ is

$$
a(x)=\exp \left(-\int_{0}^{x}\left(\left[2 \kappa(s) \tau^{1 / 2}(s)\right]^{-1}-\alpha(s)\right) d s\right)
$$


and $\zeta=\zeta(x)$, the propagation time of a wavefront passing from $x=0$ to $x$ is

$$
\zeta(x)=\int_{0}^{x} c^{-1}(s) d s
$$

Thus solutions of the first order system of partial differential equations (30) and (31) are continuous along the characteristic curves associated with the system, but may be discontinuous across these curves. From $(30)$ it is seen that the characteristic traces are $t=$ constant for $G^{+}$, and as $G^{+}(0, t)$ is certainly continuous for all $t>0$, it follows $G^{+}$ is continuous in the region $\{0<x<\ell, 0<t<\infty\}$. However examination of (34) shows that any discontinuity in $c$ or $\gamma$ will be propagated along the characteristic of (31). The conditions imposed on these functions in $\S 2$ ensure $G^{-}(x, 0)$ is continuous except possibly at $x=\ell$ where it has a discontinuity of magnitude ${ }^{4}$

$$
\left[G^{-}\right](\ell, 0)=\frac{1}{2} c(\ell) \gamma(\ell) a(\ell)
$$

in the direction of increasing $t$. This jump in $G^{-}$will propagate along the characteristic curves of $G^{-}$and so the jump across the characteristic trace passing through $(\ell, 0)$ is

$$
\left[G^{-}\right]_{x}=\frac{1}{2} c(\ell) \gamma(\ell) a(\ell) \exp \left[\int_{\ell}^{x}\left(\frac{1}{2 \kappa \sqrt{\tau}}+\delta\right) d s\right] .
$$

When the material parameters are homogeneous the systems $(30)-(31)$ or (2), or equivalently (3) or (5) can be solved exactly; see [11], ( pages 856-869) for the solution appropriate to the second order equations. The solution for the field $u$ within a semi-infinite slab, where $u$ stands for either the temperature $T$ or flux $q$ can be shown to be

$$
u(x, t)=e^{-\frac{x}{2 \kappa \sqrt{\tau}}} u\left(0, t-c^{-1} x\right)+\frac{x}{2 \kappa \sqrt{\tau}} \int_{c^{-1} x}^{t} e^{-\frac{s}{2 \tau}} \frac{\mathrm{I}_{1}\left(\frac{1}{2 \tau} \sqrt{s^{2}-c^{-2} x^{2}}\right)}{\sqrt{s^{2}-c^{-2} x^{2}}} u(0, t-s) d s,
$$

with boundary condition $u(0, t) \equiv 0, t<0$. The first part of this solution on the righthand-side of (36) represents the hyperbolic wave that travels into the medium undistorted but with attenuation. From this part of the solution it can be seen that the distance into the medium, in which the leading edge of the wave travelling twice this distance is attenuated by $e^{-1}$, the so-called e-fold distance [19], is $x_{e}=\kappa \sqrt{\tau}$. The e-fold distance appears directly in our equations (30)-(31) with obvious interpretation. The second part of the solution represented by the convolutional integral is directly representative of the dissipative or diffusive term due to the fact that the diffusivity is finite. Further discussion on the interpretation of this equation can be found in $\S 9$.

Comparison of (36) with equations (30)-(31) shows that, when the material parameters are homogeneous, $G^{-} \equiv 0$ and $G^{+}$is given by the kernel of the convolutional term. $G^{+}$is entirely due to the finite value of the diffusivity $\kappa$ for this case and can be considered as the parabolic part of the solution. The first term in (28) can then be considered as the hyperbolic part.

\footnotetext{
${ }^{4}$ Because of previous assumptions only $\gamma$ has a jump at $x=\ell$.
} 


\section{The Inverse Problem and the Reflection Operator}

We now invoke invariant imbedding to obtain functional differential equations for reflection integral operator. To this end consider the problem of scattering from the slab of thermal material of thickness $[x, \ell]$. By the Duhamel integral principle it is possible to define a reflection operator $\mathbb{R}$, where this operator is an integral operator mapping the thermal right propagating wave $v^{+}$into the left propagating wave $v^{-}$. The integral form of the reflection operators is given by

$$
v^{-}(x, t)=\mathbb{R} v^{+}=\int_{0}^{t} R(x, t-s) v^{+}(x, s) d s .
$$

We shall consider $\ell$ fixed and let the slab width $[x, \ell]$ vary continuously between 0 and $\ell$; as such there is a homotopy map from the slab of zero thickness to one of thickness $[0, \ell]$ appropriate for the problem under consideration here. This idea will convert a mixed initial/boundary value problem similar to that for the Green operators into a pure initial value problem. So the $x$ in $R(x, t)$ provides the continuous homotopy.

Insertion of (37) into (19) will show this matrix reflection kernel $R$ satisfies the following integro-differential Riccati equation

$$
\partial_{x} R-2 c^{-1} \partial_{t} R=\frac{1}{\kappa \sqrt{\tau}}[R * L-R]-[\alpha-\delta] R-R * \beta R
$$

with initial condition

$$
\gamma+2 c^{-1} R(x, 0)=0
$$

This equation will enable the solution of the inverse problem by layer stripping. It can of course also be used to solve the direct problem of finding the reflection of a wave from the slab.

\section{Discretisation of the Functional Equations}

It is usual to convert equations similar to the two previous sections to travel time coordinates, prior to attempting numerical solution. However this is not necessary as is shown in [10]. This is because the method of characteristics can still be employed in a straightforward manner; as then, for the direct problem, the characteristic traces can be conveniently integrated prior to attempting to solve the equations. On using the notation of [2] a parametric equation for the characteristic trace of equation (31) can be written as

$$
t=\tau^{-}(s ; x, t)
$$

where $\left(s ; \tau^{-}(s ; x, t)\right)^{5}$ describes a curve in $\mathbb{R}^{2}$ passing through $(x, t)$ and $s$ being a parameter on the $x$-axis. For equation (31) the characteristic traces are translates of each other (as explained in Appendix $\mathrm{C}$ of [2] this corresponds to an area preserving flow). This can be seen in Figure 2 This is because the wave speed does not depend upon $t$. It suffices here then, to just consider the trace passing through $(\ell, 0)$. The characteristic

\footnotetext{
${ }^{5}$ There should not be any confusion with the symbol for the characteristic curves and that for the relaxation time, $\tau(x)$, as the former has superscripts and three arguments.
} 
trace corresponding to the case when the sign in the principal part of (31) is positive is written as $\tau^{+}(s ; x, t)$, and it has positive slope. The trace passing through $(0,0)$ can then be written as

$$
t=\tau^{+}(s ; 0,0)=\widetilde{\zeta}(s)
$$

where $\tilde{\zeta}$ is given by

$$
\widetilde{\zeta}=2 \int_{0}^{x} c^{-1}(s) d s
$$

Note the relationship to (35).

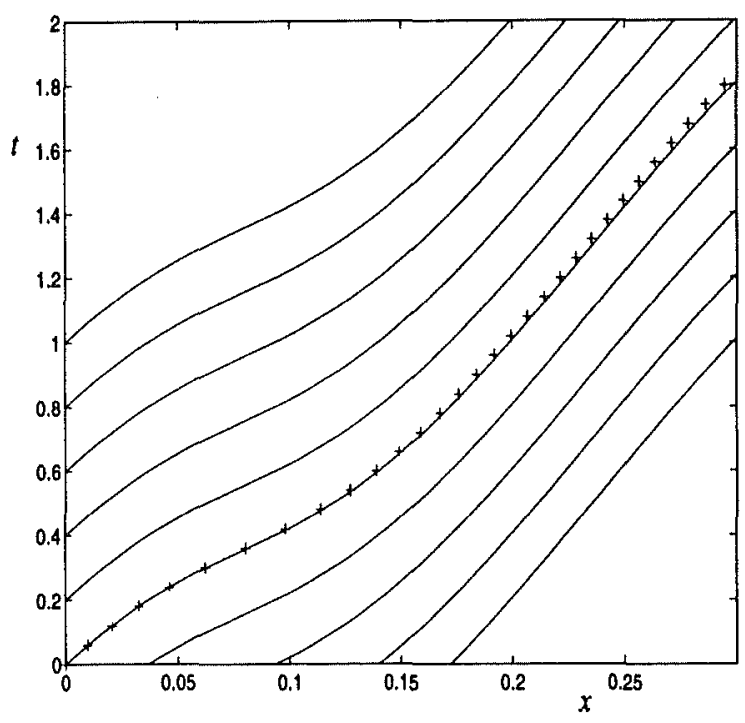

FIGURE 2. Reconstruction of a characteristic trace by the inverse algorithm. Several characteristic traces $\tau^{+}(s ; x, t)$ are shown together with the reconstructed values + from the inverse algorithm discussed in $\S 9$ when $N=2^{5}$ for slowness (57) with $A=-0.75$.

Appropriate points on the characteristic traces may now be determined numerically. First define the natural numbers $i, j, N \in \mathbb{N}$, then a mesh $\left\{x_{i}\right\}_{i=0}^{N}$ with uniform mesh interval $h=\ell / N$ and $x_{0}=0, x_{i}=x_{i-1}+h, \quad 1 \leq i \leq N$ is established. The non-uniform mesh points $t_{i}=\widetilde{\zeta}\left(x_{i}\right)$ can then be evaluated by numerical quadrature of (40) using the trapezoid rule. The time $t_{N}=\mathrm{T}=\widetilde{\zeta}\left(\mathrm{x}_{\mathrm{N}}\right)$, which is the return travel time taken by a wavefront to travel from $x=0$ to $x=\ell$ and back again, is important for our subsequent development. Our algorithm is more easily implemented with a uniform $t$-mesh, however this can be done by finding the inverse function to $\widetilde{\zeta}$. We estimate this function by inverse 
interpolation of $\widetilde{\zeta}\left(x_{i}\right)$ using a clamped cubic spline interpolation ${ }^{6}$ and then subsequent evaluation of the resultant fourth order approximate to $x_{i}=\widetilde{\zeta}^{-1}\left(t_{i}\right)$, where now $\left\{t_{i}\right\}_{i=0}^{N}$ with $p=\mathrm{T} / \mathrm{N}$ and $t_{0}=0, t_{i}=t_{i-1}+p, \quad 1 \leq i \leq N$ is a uniform mesh on the $t$-axis; whereas the mesh $\left\{x_{i}\right\}_{i=0}^{N}$ is now non-uniform. The non-uniform step size on the $x$-mesh is $h_{i-1}=x_{i}-x_{i-1}, \quad 1 \leq i \leq N$. The existence of the inverse function $\widetilde{\zeta}^{-1}$ is assured by the inverse function theorem and our requirement that $c>0$. Points on the characteristic trace are then represented as $\left\{x_{i}, t_{i}\right\}_{i=0}^{N}$ and integration of the functional equations, for any problem in which $c(x)$ is known, by the method of characteristics is straightforward.

8.1. Green Function equations. For notational convenience we first rewrite (31) as

$$
\partial_{x} G^{-}-2 c^{-1} \partial_{t} G^{-}=F^{(2)}\left(x, G^{+}(x, t), G^{-}(x, t)\right),
$$

where $F^{(2)}$ is the linear function of $G^{ \pm}$as expressed by the left-hand-side of (31). For the direct problem all the material parameters are known, and it is desired to evaluate the field within and outside the slab. Direct integration along a characteristic of equation (31), from $\left(x_{i+1}, t_{j-1}\right)$ to $\left(x_{i}, t_{j}\right)$ yields the result

$$
\begin{aligned}
G^{-}\left(x_{i}, t_{j}\right)-G^{-} & \left(x_{i+1}, t_{j-1}\right) \\
= & -\int_{x_{i+1}}^{x_{i}} F^{(2)}\left(s, G^{+}\left(s, \tau^{-}\left(s ; x_{i+1}, t_{j-1}\right)\right), G^{-}\left(s, \tau^{-}\left(s ; x_{i+1}, t_{j-1}\right)\right)\right) d s .
\end{aligned}
$$

The characteristic traces for $(30)$ are parallel to the $x$-axis and so this equation can be integrated from $\left(x_{i-1}, t_{j}\right)$ to $\left(x_{i}, t_{j}\right)$ to obtain

$$
G^{+}\left(x_{i}, t_{j}\right)-G^{+}\left(x_{i-1}, t_{j}\right)=\int_{x_{i-1}}^{x_{i}} F^{(1)}\left(s, G^{+}\left(s, t_{j}\right), G^{-}\left(s, t_{j}\right)\right) d s,
$$

where $F^{(1)}$ is the linear function of $G^{ \pm}$as expressed by the left-hand-side of $(30)$. Up to this point, these are exact results without approximations. Denote by

$$
\begin{gathered}
G_{i, j}^{ \pm}=G^{ \pm}\left(x_{i}, t_{j}\right)=G^{ \pm}\left(x_{i}, j p\right), \\
\alpha_{i}=\alpha\left(x_{i}\right), \quad \beta_{i}=\beta\left(x_{i}\right), \quad \gamma_{i}=\gamma\left(x_{i}\right), \quad \delta_{i}=\delta\left(x_{i}\right), \\
\kappa_{i}=\kappa\left(x_{i}\right), \quad \tau_{i}=\tau\left(x_{i}\right), \quad k_{i}=k\left(x_{i}\right) \\
a_{i}=a\left(x_{i}\right)=\exp \left(-\int_{0}^{x_{i}}\left(\left[2 \kappa(s) \tau^{1 / 2}(s)\right]^{-1}-\alpha(s)\right) d s\right), \\
\left(L * G^{ \pm}\right)_{i, j}=\int_{0}^{t_{j}} L\left(x_{i}, t_{j}-s\right) G^{ \pm}\left(x_{i}, s\right) d s, \\
L_{i, j}=L\left(x_{i}, t_{j}\right)
\end{gathered}
$$

\footnotetext{
${ }^{6}$ The end boundary conditions for the clamped spline are found by using cubic interpolants at each of the endpoints $x=0$ and $x=\ell$, and then using the first derivative of these interpolants to estimate the end derivatives of $\widetilde{\zeta}$.
} 
so that the column vector $\mathbf{F}$ for the differential system becomes

$$
\begin{aligned}
F_{i, j}^{(1)} & =F^{(1)}\left(x_{i}, G^{+}\left(x_{i}, t_{j}\right), G^{-}\left(x_{i}, t_{j}\right)\right) \\
& =\frac{1}{2 \kappa_{i} \sqrt{\tau_{i}}}\left(a_{i} L_{i, j}+\left(L * G^{+}\right)_{i, j}-G_{i, j}^{+}\right)+\alpha_{i} G_{i, j}^{+}+\beta_{i} G_{i, j}^{-} \\
F_{i, j}^{(2)} & =F^{(2)}\left(x_{i}, G^{+}\left(x_{i}, t_{j}\right), G^{-}\left(x_{i}, t_{j}\right)\right) \\
& =\frac{1}{2 \kappa_{i} \sqrt{\tau_{i}}}\left(G_{i, j}^{-}-\left(L * G^{-}\right)_{i, j}\right)+\delta_{i} G_{i, j}^{-}+\gamma_{i} G_{i, j}^{+}
\end{aligned}
$$

for $i=0,1,2, \ldots, N$ and $j=0,1,2, \ldots, N$. We shall need both explicit and implicit type algorithms which can readily be derived from the preceding two equations, (42) and (43), by simply using the rectangular or trapezoidal quadrature rules, in approximating the integrals for the right-hand-side in these equations, respectively. The convolutional terms in these equations are estimated by the trapezoidal rule as

$$
(L * G)_{i, j}=p \sum_{n=0}^{j} " L_{i, j-n} G_{i, n},
$$

where the double prime on the summation sign signifies that the first and last term in the summation is to be halved. The trapezoidal rule is also utilised in approximating the attenuation $a_{i}$, with an algorithm similar to that presented later for the initial value of $G^{+}$ (Note the $x$-mesh is non-uniform and see equation (45)).

The explicit Euler characteristic rule is

$$
\begin{aligned}
& G_{i, j}^{+}=G_{i-1, j}^{+}+h_{i-1} F_{i-1, j}^{(1)}, \quad i>0, \quad j>0 \\
& G_{i, j}^{-}=G_{i+1, j-1}^{-}-h_{i} F_{i+1, j-1}^{(2)}, \quad i \geq 0, \quad j>0
\end{aligned}
$$

and the implicit trapezoidal characteristic rule is

$$
\begin{aligned}
& G_{i, j}^{+}=G_{i-1, j}^{+}+\frac{h_{i-1}}{2}\left(F_{i-1, j}^{(1)}+F_{i, j}^{(1)}\right), \quad i>0, \quad j>0 \\
& G_{i, j}^{-}=G_{i+1, j-1}^{-}-\frac{h_{i}}{2}\left(F_{i+1, j-1}^{(2)}+F_{i, j}^{(2)}\right), \quad i \geq 0, \quad j>0 .
\end{aligned}
$$

In wave splitting problems, investigators often utilise the linearity of the Green function equations to solve explicitly the implicit equations. This complicates the computer algorithm. Our algorithms use the conventional predictor-corrector approach by using first the explicit rule to estimate $G_{i, j}^{ \pm}$and then to utilise the implicit system in conventional fixed point iteration. Convergence is assured for sufficiently small $p$, with only a few iterations. This approach, although simplifying the computational algorithm has its disadvantages in the limit as the equations become more closely to parabolic, that is if the relaxation parameter is very small. Then the differential equations become stiff, and fixed point iteration is not appropriate unless $p$ is very small. With parabolic equations, the computational requirements are generally that the equations are integrated to equilibrium as fast as possible; this implies that $p$ should not be small. However with hyperbolic equations, the wave 
propagation effects are important, and then small $k$ values must be employed to provide sufficient resolution of the wave details. As our main concern is with wave effects in this paper we shall not consider this point further.

The computational cost of the convolutional terms (44) is high, when $t$ is large, so care needs to be taken in the iterative loop not to recalculate the full convolution. The evaluation of the convolutional terms can be performed in an efficient manner using the discrete Fourier transform, but we shall not consider this here. Examination of Figure 3 shows the geometric structure of the computational molecule for the implicit rule and this illustrates that careful consideration of the computation of the convolution terms can reduce the computational cost. The initial values for the Green functions at $x=0$ are
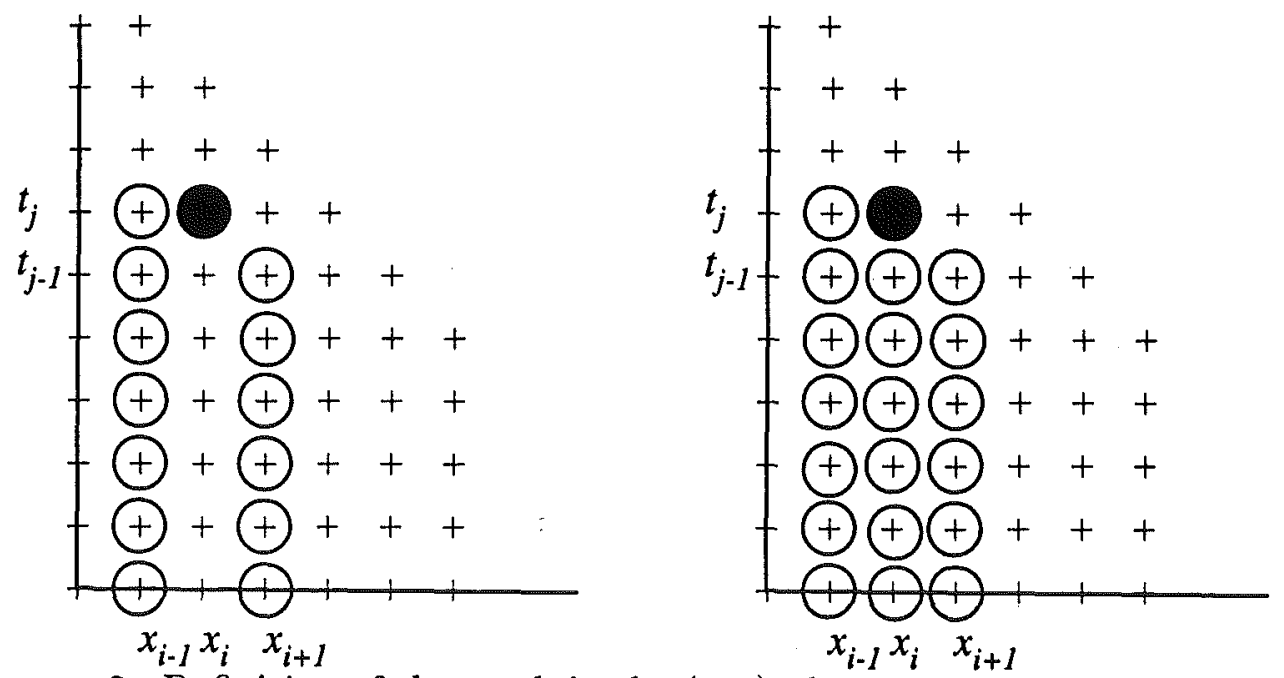

FIGURE 3. Definition of the mesh in the $(x, t)$-plane and the computational module of the algorithm based upon the Green functions equations.

(a) The explicit algorithm

(b) The implicit algorithm

$$
G_{i, 0}^{-}=-\frac{1}{2} c_{i} \gamma_{i} a_{i}, \quad 0 \leq i \leq N
$$

and by defining

$$
g_{i}=\frac{a_{i}}{4} h_{i-1}\left(f_{i}+f_{i-1}\right), \quad \text { with } f_{i}=\left(\frac{1}{4 \kappa_{i} \tau_{i}^{3 / 2}}-\gamma_{i} \beta_{i} c_{i}\right)
$$

the remaining initial values are

$$
G_{i, 0}^{+}=G_{i-1,0}^{+}+g_{i}, \quad 1 \leq i \leq N,
$$

where again the trapezoidal rule has been used to discretise (33). The boundary values of the Green function at $x=0$ are

$$
\begin{aligned}
G_{0, j}^{+} & =0, \\
G_{0, j}^{-} & =R_{0, j},
\end{aligned}
$$


where the values of the discretised reflection kernel $R_{0, j}=R(0, j p), \quad 0 \leq j \leq N$ are the sought quantities in the direct algorithm - as then the reflected wave may be calculated through (37). The algorithm starts from the lower left corner of the mesh depicted in Figure 3, from the horizontal line $t=0$, where the initial values are known and the vertical line $x=0$ where the boundary values are known, and proceeds to higher $j$-values, on each line $j=$ constant calculating the Green functions from the left to the right. If the total field is required for a given incident field equations (27) and (28) are used.

8.2. Reflection kernel direct problem. For notational convenience we first rewrite (38) as

$$
\partial_{x} R-2 c^{-1} \partial_{t} R=F(x, R(x, t)),
$$

where $F$ is a non-linear function of $R$, as expressed by the left-hand-side of (38). For the direct problem all the material parameters are known, and it is desired to evaluate the reflection kernel at $x=0$. Direct integration along a characteristic of equation (46), from $\left(x_{i+1}, t_{j-1}\right)$ to $\left(x_{i}, t_{j}\right)$ yields the result

$$
R\left(x_{i}, t_{j}\right)-R\left(x_{i+1}, t_{j-1}\right)=-\int_{x_{i+1}}^{x_{i}} F\left(s, R\left(s, \tau^{-}\left(s ; x_{i+1}, t_{j-1}\right)\right)\right) d s .
$$

with

$$
F_{i, j}=\frac{1}{\kappa_{i} \sqrt{\tau_{i}}}\left((R * L)_{i, j}-R_{i, j}\right)-\left[\alpha_{i}-\delta_{i}\right] R_{i, j}-\beta_{i}(R * R)_{i, j}
$$

The equation (47) is now discretised, by similar methods to the last section, to yield the explicit and implicit difference equations

$$
\begin{aligned}
& R_{i, j}=R_{i+1, j-1}-h_{i} F_{i+1, j-1}, \quad i \geq 0, \quad j>0, \\
& R_{i, j}=R_{i+1, j-1}-\frac{h_{i}}{2}\left(F_{i+1, j-1}+F_{i, j}\right), \quad i \geq 0, \quad j>0,
\end{aligned}
$$

respectively. The $R * L$ kernel convolutional term is treated as (44), but replacing $G$ by $R$, and the quadratic convolutional term is discretised as

$$
(R * R)_{i, j}=p \sum_{n=0}^{j "} R_{i, j-n} R_{i, n}
$$

by the trapezoidal rule. Figure 4 illustrates the geometry of the computational stencil. The initial values of $R_{i, j}$ are

$$
R_{i, 0}=-\frac{\gamma_{i} c_{i}}{2}, \quad 0 \leq i \leq N .
$$

The values of the discretised reflection kernel $R_{0, j}, \quad 0 \leq j \leq N$ are the sought quantities in the direct algorithm. The algorithm starts from the lower left corner from the horizontal line $t=0$, where the initial values are known and proceeds to higher $j$-values, diagonally calculating the reflection kernel from the left to the right. As for the Green functions, the explicit and implicit algorithms are used in predictor-corrector mode, with convergence assured for small enough $p$. 

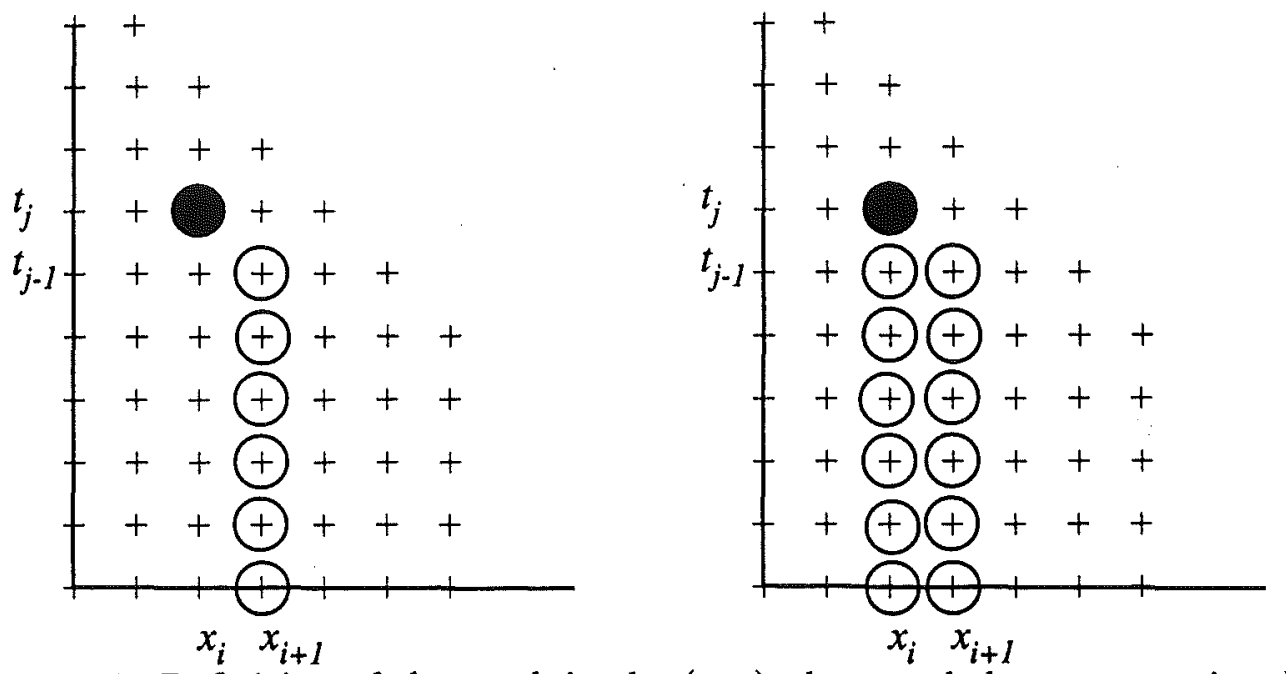

FIGURE 4. Definition of the mesh in the $(x, t)$-plane and the computational module of the algorithm based upon the reflection kernel equation.

(a) The explicit algorithm

(b) The implicit algorithm

8.3. Reflection kernel inverse problem. The inverse algorithm is based on the discretised imbedding equation, see (48) and (49), as is the direct problem, but because the equations will now be integrated in the direction of increasing $x$ the sign of the term which is multiplied by $h_{i}$, in both these equations, must be inverted, so giving

$$
\begin{aligned}
& R_{i, j}=R_{i+1, j-1}+h_{i} F_{i+1, j-1}, \quad i>0, \quad j \geq 1, \\
& R_{i, j}=R_{i+1, j-1}+\frac{h_{i}}{2}\left(F_{i+1, j-1}+F_{i, j}\right), \quad i>0, \quad j \geq 1 .
\end{aligned}
$$

In our original model described by equation (2) there are five material functions. It is apparent only one of these functions can be reconstructed from the one given measurement set, the reflection kernel. We will therefore restrict our considerations to only reconstructing the wave slowness. Then depending upon the problem, either $\tau$ or $\kappa$ can be recovered with the assumption that the other is known; that is only one function can be recovered by our method. For simplicity in the sequel we make the choice $\tau=$ constant , $0<x<\ell$, and assumed to be known. In our inverse algorithm the discrete values of the reflection kernel $R$ are assumed to be known from a scattering experiment, and the goal in the inverse algorithm is to retrieve the slowness $c_{i}^{-1}$. The slowness is easily recovered once the values of $R$ on the horizontal line $t=0$ have been calculated, as

$$
c_{i}=-2 R_{i, 0} / \gamma_{i}
$$

The computations start from the initial value $R_{0, j}, \quad 0 \leq j \leq N$ and proceeds from the lower left corner in a diagonal direction down the characteristic traces to the line $t=0$; computing the value of $c_{i}$ from (53) when $t=0$. See Figure 4 . It remains for us to describe how the algorithm is implemented as the curved characteristics are not known a priori 
because $c(x)$ is initially unknown. This means that when the method of characteristics is employed, the $x_{i}$ are also initially unknown. The actual determination of the slowness is carried out in the integration step from $t_{1}=p$ to $t_{0}=0$.

We describe the algorithm to determine $x_{i}, c_{i}$ from $\left(x_{i-1}, t_{1}\right), t_{1}=p$, and all other material parameters and values of $R_{i-1, p}$. Determination of $R_{i, j}, 1 \leq j \leq n$ when $c_{i}$ is known is similar to the direct algorithm except the integration is carried out from left to right.

From the equation for $\widetilde{\zeta}$, equation (40)

$$
t_{i}-t_{i-1}=2 \int_{x_{i-1}}^{x_{i}} c^{-1}(s) d s
$$

the next value of $x$ on the characteristic trace, $x_{i}$ can be estimated by use of either the rectangular or trapezoidal quadrature rule approximants. Equations (22) - (24), together with the fact that for the problem considered here $e \equiv 0$, implies

$$
\frac{d c}{d x}=4 R(x, 0)+c \frac{d \ln (k)}{d x}
$$

for the system (2). For both second order equations (3) and (5) the last term is zero, as it is for the system case, if $k \neq k(x)$. For simplicity we assume that this is true in what follows. Use of the trapezoidal quadrature formula then yields the discretised formula

$$
c_{i}=c_{i-1}+2 h_{i-1}\left(R_{i, 0}+R_{i-1,0}\right) .
$$

This means to solve the inverse problem at the ultimate integration step of equation (52) from $t=p$ to $t=0$, there are three non-linear equations required to be solved. These are (54) (55) (52), so yielding on solution $x_{i}, c_{i}$ and $R_{i, 0}$ respectively. We solve this system by fixed point iteration, and again it is possible to prove convergence of this method provided that $p$ is sufficiently small. In order to obtain rapid convergence we use an initial estimate of the solution obtained from the an appropriate explicit form of discrete approximations to the aforementioned equations. These can be straightforwardly shown to be given by $(51),(56)$ together with the explicit form of (54)

$$
x_{i}=x_{i-1}+\frac{\left(t_{i}-t_{i-1}\right) c_{i-1}}{2}, \quad \text { or } h_{i-1}=\frac{p c_{i}}{2} .
$$

The fixed point iteration is carried out with the implicit form of these equations which are (52)and (56) together with

$$
x_{i}=x_{i-1}+\frac{\left(t_{i}-t_{i-1}\right)}{\left(c_{i-1}^{-1}+c_{i}^{-1}\right)} .
$$

One final complication is that, as for the direct problem, equation (52) is also solved by iteration. 

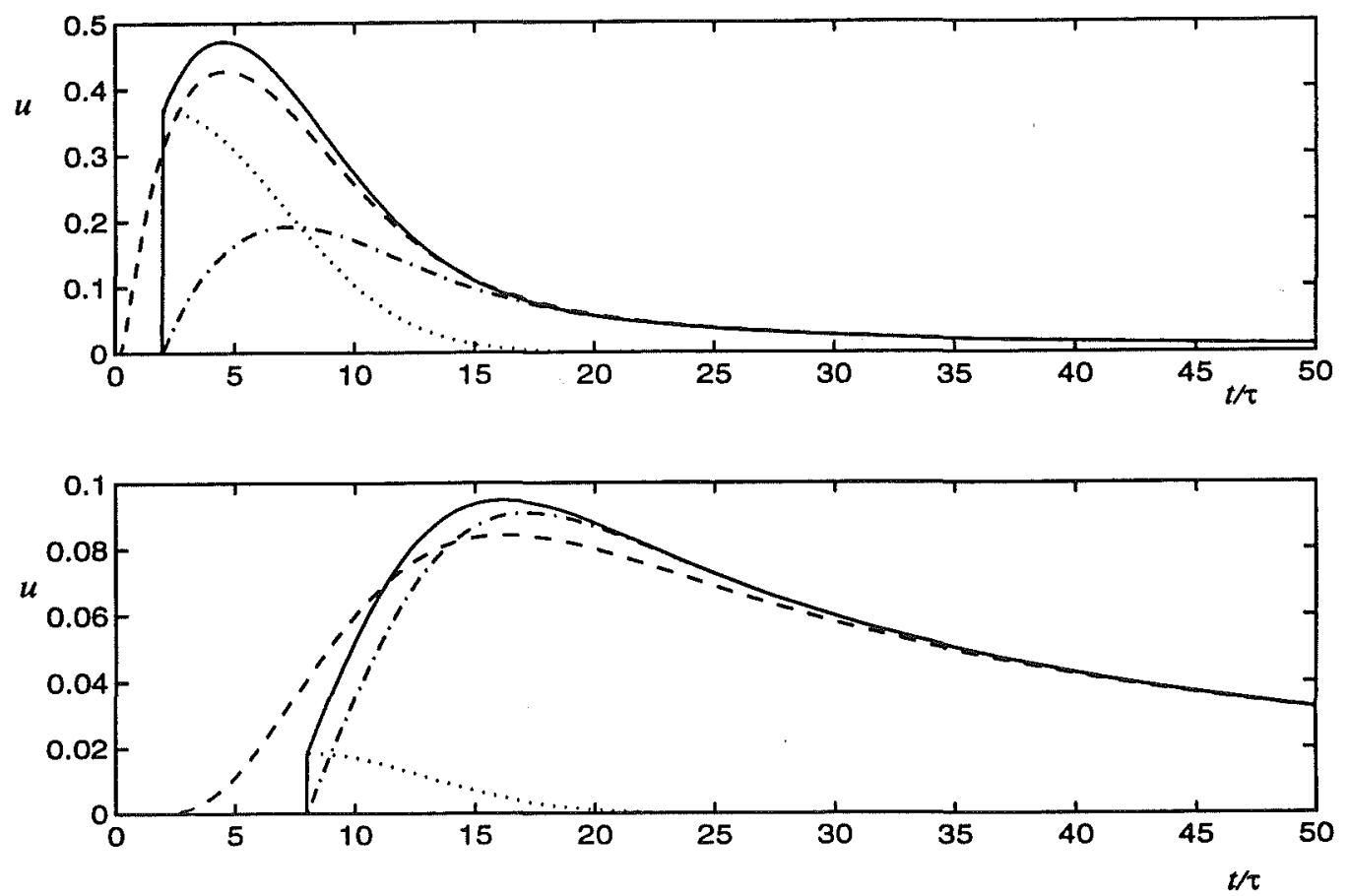

Figure 5. The internal field, for the direct problem, at a point in a homogeneous semi-infinite region.

(a) Fields at $2 x_{e}$ when $\kappa=0.1, \tau=0.1$,

- Total field, - - - Parabolic part of total field, .. Hyperbolic part of total

field, - - Solution of non-causal parabolic problem.

(b) Fields at $8 x_{e}$ when $\kappa=0.1, \tau=0.1$,

- Total field, - - - Parabolic part of total field, ... Hyperbolic part of total field, - - Solution of non-causal parabolic problem.

\section{Numerical EXAMPLES}

The field generated inside a semi-infinite homogeneous slab with a exponentially decaying pulse $^{7}$ that is incident upon the face $x=0$ is first analysed. In Figure 5(a) and (b) we show the total field at interior points that are twice and eight times the e-fold distance, that is $2 x_{e}$ and $8 x_{e}$, respectively. It can be observed that the wave field takes a finite time to reach the internal point. Note the non-causal solution to the parabolic problem $(\tau=0)$ has been superimposed on these figures. The convergence of the hyperbolic solution to this solution, after the initial transient propagating pulse has past, can be easily observed. The time scale of the figures has been normalised by the time taken for the wavefront to travel a distance of $x_{e}$; which is $\tau$. Observe that the field on the leading edge of the hyperbolic

\footnotetext{
${ }^{7}$ The boundary condition taken for solution of the direct problems in this paper was taken as $u(0, t)=$ $\exp \left(-2.0 t^{2}\right), t>0$. Note the lack of dependence on $\tau$, this has the effect of making the boundary condition more slowly varying with respect to time when the relaxation time is smaller.
} 
part of the solution is attenuated by $e^{-1}$, for the case when the field is evaluated at $2 x_{e}$. Also superimposed on these figures are the component parts of the solution as discussed in $\S 6$.

Figure 6 shows the total field at the point $2 x_{e}$, but with a different time scales introduced by reducing the value of the relaxation time by two orders of magnitude. It is observed that the field approaches that of the parabolic model, after the wave front has reached the point, quite rapidly when $\tau<<1$, reference [12] predicts that this convergence of the causal solution to non-causal one occurs typically in time periods of $\mathcal{O}(\tau)$. It is important to note however, that the incident field, taken here, is successively more slowly varying as $\tau$ decreases and that even for small values of relaxation time if the incident pulse varies rapidly with respect to $\tau$ the solution of the hyperbolic equation will deviate significantly from that of the parabolic equation until the transient wave effects have decayed. This means that around the arrival time of the wave the solution will look like figure 5. The numerical results depicted in these figures were computed with the Green function algorithm, and the results could not be distinguished in these figures from computations on the analytical result (36). The shape of the Figures $5-6$ are independent of $\kappa$ because this parameter
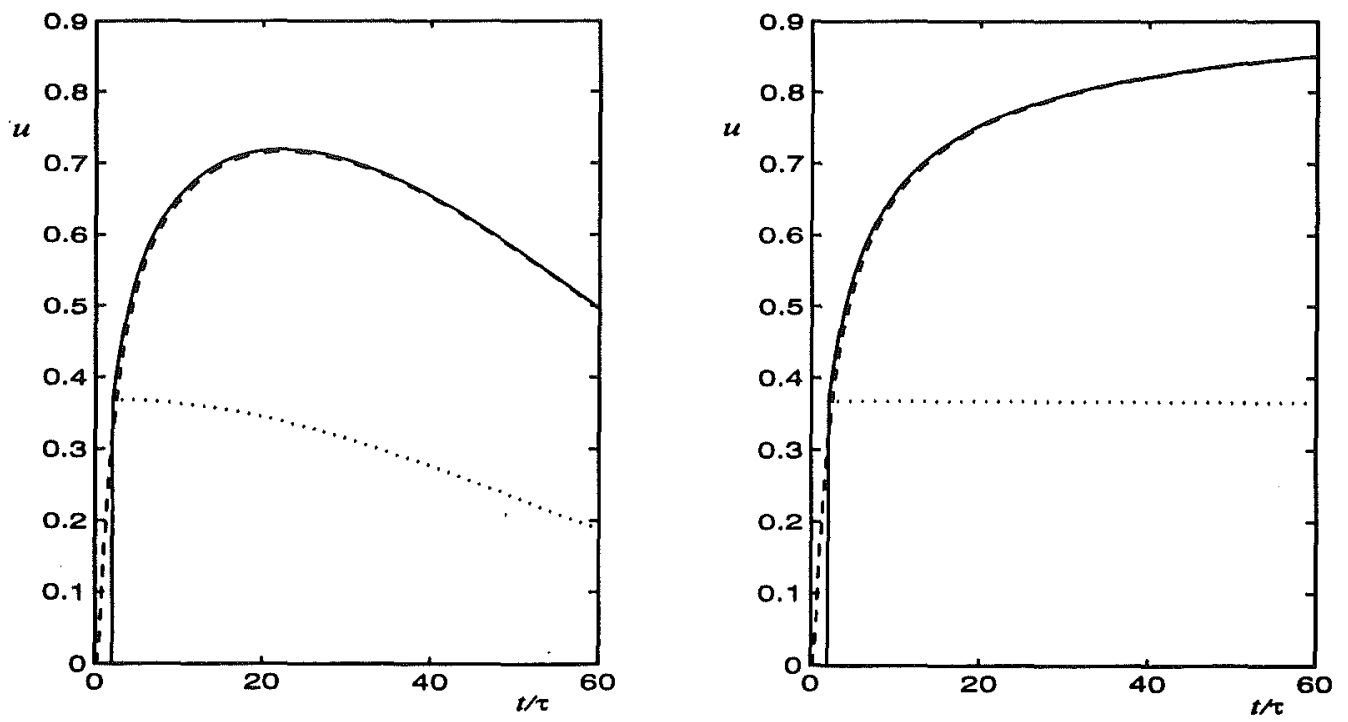

FIguRE 6. The internal field, for the direct problem, at a point in a homogeneous semi-infinite region.

(a) Fields at $2 x_{e}$ when $\kappa=0.1, \tau=0.01$,

- Total field, ... Hyperbolic part of total field, - - Solution of non-causal parabolic problem.

(b) Fields at $2 x_{e}$ when $\kappa=0.1, \tau=0.001$,

- Total field, ... Hyperbolic part of total field, - - Solution of non-causal parabolic problem.

changes both the the e-fold depth $x_{e}$ and the wave speed. 
Figure 7 and Figure 8 show the field inside a finite slab with a spatially varying slowness

$$
c^{-1}(x)=0.1\left(1+A \sin \left(2 \pi x /\left(10 x_{e}\right)\right)^{-1 / 2}, \quad \text { with } A=0.75 .\right.
$$

This slowness profile is utilised in numerical experiments later in this paper and is illustrated in Figures 9-10. We note that the time is normalised as previously, but now as the material parameters are spatially varying we chose the initial value of the material parameters to define an average e-fold distance, that is $x_{e}=\kappa(0) \sqrt{\tau(0)}$. This accounts for the fact, as observed from the figures that the wavefront reaches the observation point in a shorter time than predicted if the medium was homogeneous. From these figures note the smaller contribution from the hyperbolic part of the traveling wave as the penetration depth gets larger. In figure Figure 8(a) the total field and $u^{+}$cannot be distinguished from each other to the scale of our figure. Comparison should be made with these figures and
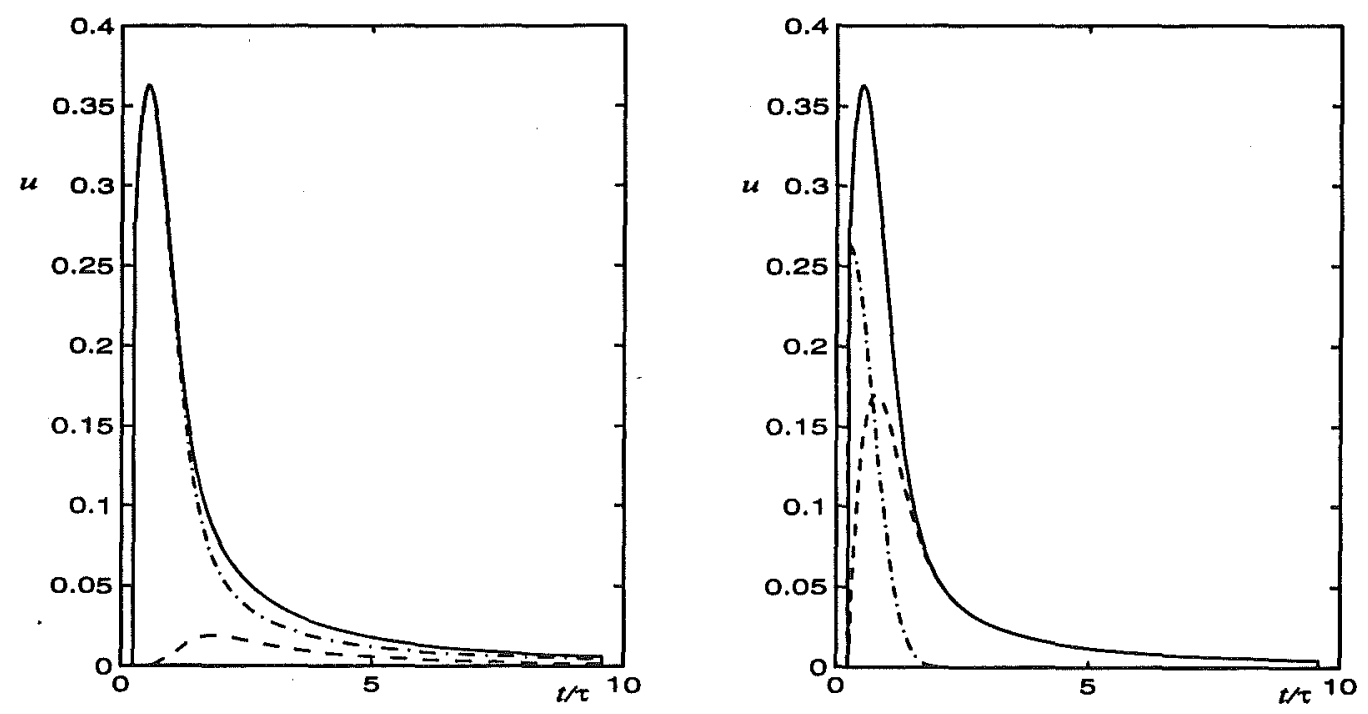

FIgure 7 . The internal field of the direct problem with spatially varying slowness in a slab of width $\ell=10 x_{e}$ at a point $2 x_{e}$ when $\tau(0)=0.1, x_{e}=$ $\kappa(0) \sqrt{\tau(0)}$, and $\kappa(0)=0.1\left(1+0.75 \sin \left(2 \pi x /\left(10 x_{e}\right)\right)\right)^{-1 / 2}$

(a) - Total field, $-\cdots u^{+},----u^{-}$,

(b) $-u^{+},--$parabolic part of $u^{+},-\cdot-$ hyperbolic part of $u^{+}$.

Figure 5.

We generate the reflection kernel required as the initial data to test the inverse algorithm by one of the numerical algorithms developed in this paper for the direct problem. To ensure that an inverse crime is not being committed, the reflection kernel is calculated at a greater number of points than required by the inverse imbedding algorithm, namely $4(N+1)$ points, but via the Green function technique, and then a clamped cubic spline is fitted to this data. Finally the spline is used to interpolate to the appropriate mesh of $N+1$ points as required for initial data by the imbedding method. 

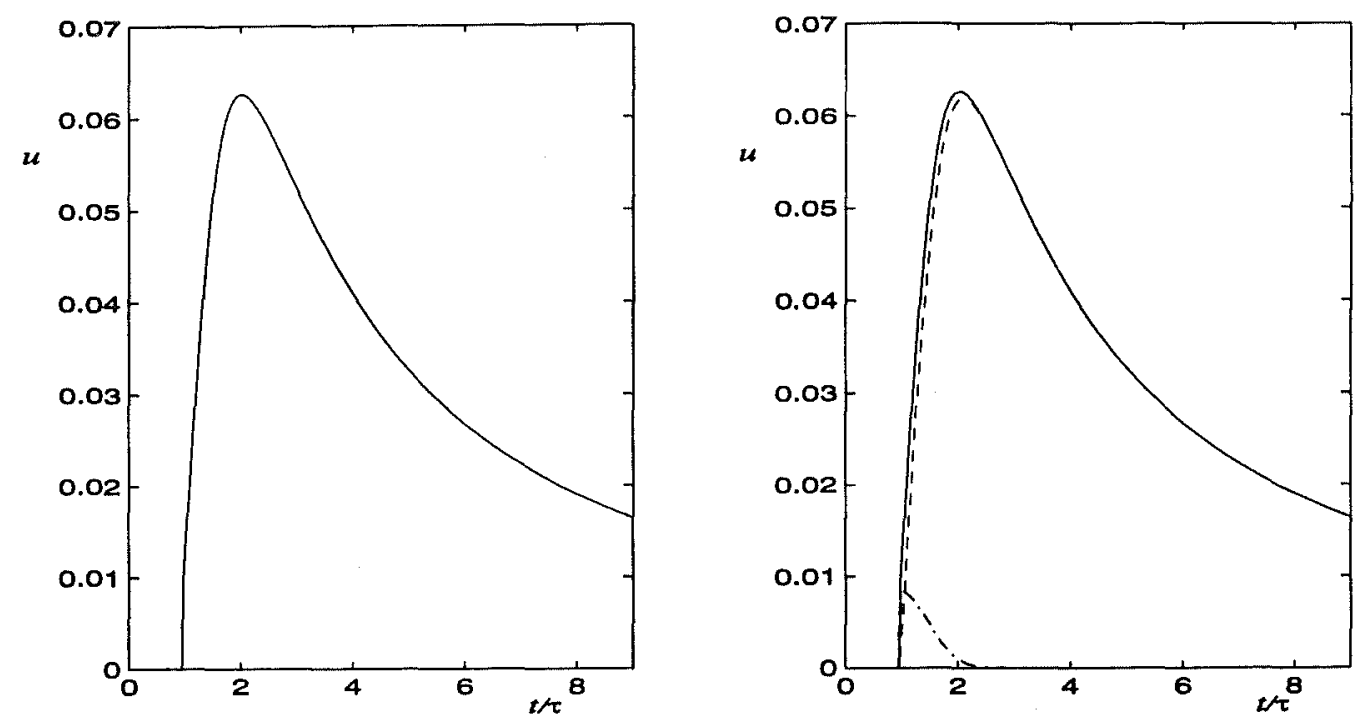

FIGURE 8 . The internal field of the direct problem with spatially varying slowness in a slab of width $\ell=10 x_{e}$ at a point $10 x_{e}$ when $\tau(0)=0.1$, $x_{e}=\kappa(0) \sqrt{\tau(0)}$, and $\kappa(0)=0.1\left(1+0.75 \sin \left(2 \pi x /\left(10 x_{e}\right)\right)\right)^{-1 / 2}$

(a) - Total field, $-\cdots u^{+},----u^{-}$,

(b) $-u^{+},--$parabolic part of $u^{+},-\cdots$ hyperbolic part of $u^{+}$.

In Figures 9-10 we illustrate the effectiveness of our algorithm by showing an exact slowness and the reconstructed slowness for one mesh size. It is seen that diffusivity in a thermal model can be reconstructed provided that the finite time of propagation of a heat wave is taken into effect. Note that the slowness can even be reconstructed as deep as ten e-fold distances inside the slab with small error, although the error increases with distance into the slab. In figure 2 the actual characteristic traces and a reconstructed trace from our algorithm are displayed.

Numerical experiments indicate that our methods have a consistency error of $\mathcal{O}\left(p^{2}\right)$ and possess an even power asymptotic discretisation expansion. Techniques similar to those used in [18] must be utilised to prove this however.

\section{SUMmaRY}

This paper builds up a general wave splitting and imbedding theory for solution of both direct and inverse problems associated with thermal processes. It is done by using a full representation of the thermal phenomenon by virtue of Cattaneo's law. This law by ensuring finite thermal propagation speeds, enables an imbedding equation to layer strip the medium; so allowing the solution of the inverse problem of determination of a spatially varying diffusivity. Although this is important in its own right for problems in which the hyperbolic nature of thermal waves must be taken into account, it also has considerable 

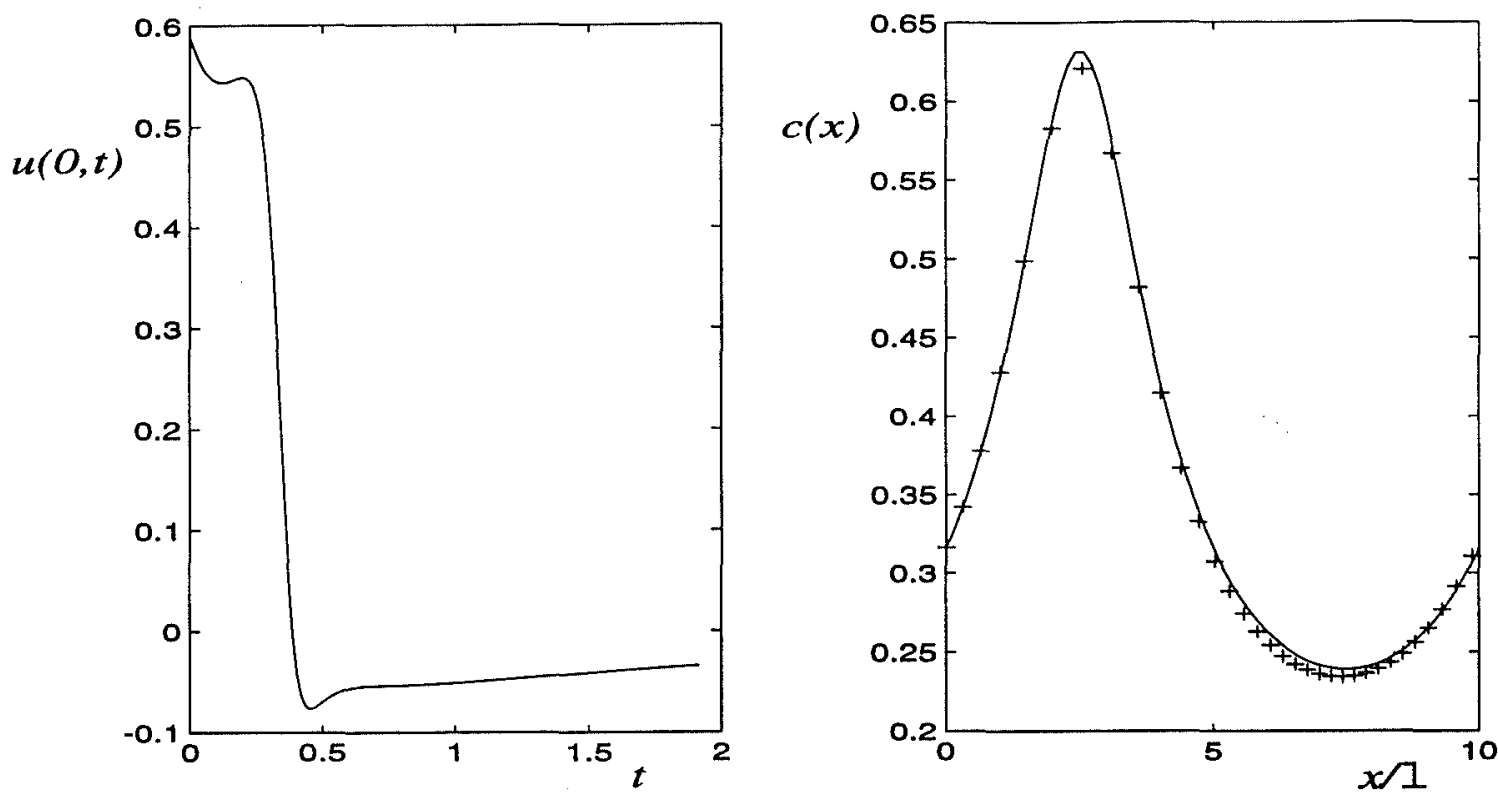

Figure 9. The solution of the inverse problem; reconstruction of slowness (57) with $A=-0.75$

(a) The reflection kernel from which the slowness was reconstructed.

(b) The exact slowness - and the reconstructed slowness + when $N=2^{5}$

impact on parabolic problems as well. Our methods can then be applied to parabolic problems, but then the wave speed parameter can be thought of as a regularisation parameter. This application is left to a later paper.

\section{ACKNOWLEDGEMENTS}

One of the authors (D.J.N.W) wishes to express his gratitude to the University of Canterbury for granting him study leave which enabled this project to be completed. The other author (P.O) has in part been supported by the Swedish Research Council for Engineering Sciences (TFR). The collaboration was carried out while (D.J.N.W) was visiting the Department of Electromagnetic Theory, University of Lund, Lund, Sweden and their hospitality is gratefully acknowledged. Finally (D.J.N.W) wishes to thank the Wenner Gren Foundation for the research grant which enabled the Lund visit, to be possible.

\section{REFERENCES}

[1] Ingegerd §̊berg, Gerhard Kristensson, and David J.N. Wall. Propagation of transient electromagnetic waves in time-varying media-direct and inverse scattering problems. Inverse Problems, 11(1):29-49, 1995.

[2] Ingegerd Åberg, Gerhard Kristensson, and David J.N. Wall. Transient waves in non-stationary media. Technical Report LUNDEDX/(TEAT-7037)/1-23/(1994), Teoretisk Elektroteknik, Tekniska Högskolan i Lund, 1995. To appear J. Math. Phys.

[3] C. Cattaneo. Sulla conduzione de calore. Atti del Semin. Mat. e Fis. Univ. Modena, 3:3, 1948. 

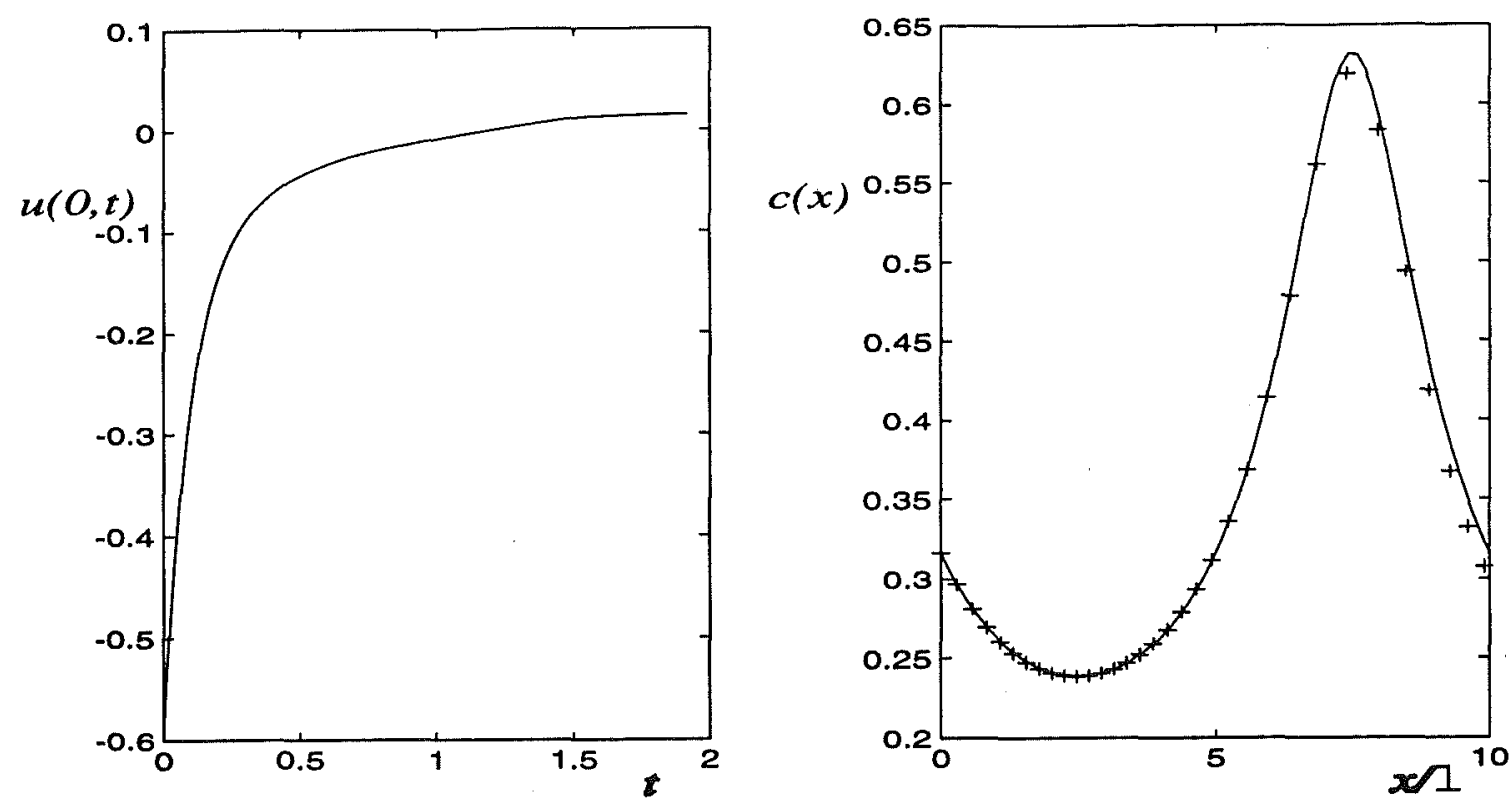

FIGURE 10. The solution of the inverse problem; reconstruction of slowness

(57) with $A=0.75$

(a) The reflection kernel from which the slowness was reconstructed.

(b) The exact slowness - and the reconstructed slowness + when $N=2^{5}$

[4] J.P. Corones, G. Kristensson, P. Nelson, and D.L. Seth, editors. Invariant Imbedding and Inverse Problems. SIAM, New York, 1992.

[5] S. He. Factorization of a dissipative wave equation and the Green function technique for axially symmetric fields in a stratified slab. J. Math. Phys., 33(3):953-966, 1992.

[6] D. D. Joseph and Luigi Preziosi. Heat waves. Rev. Mod. Phys., 61(1):41-73, 1989.

[7] D. D. Joseph and Luigi Preziosi. Addendum to the paper: Heat waves. Rev. Mod. Phys., 62(2):375$391,1990$.

[8] G. Kristensson and R.J. Krueger. Direct and inverse scattering in the time domain for a dissipative wave equation. Part 3: Scattering operators in the presence of a phase velocity mismatch. J. Math. Phys., 28(2):360-370, 1987 .

[9] R. J. Krueger and R. L. Ochs. A Green's function approach to the determination of internal fields. Wave Motion, 11:525-543, 1989.

[10] J. Lundstedt and S. He. Time-domain direct and inverse problems for a nonuniform $L C R G$ line with internal sources. IEEE Trans. Electromagn. Compatibility, 1995. (in press).

[11] P.M. Morse and H. Feshbach. Methods of Theoretical Physics, volume 1. McGraw-Hill Book Company, New York, 1953.

[12] Gabreil B. Nagy, Omar E. Oritz, and Oscar A. Reula. The behaviour of hyperbolic heat equations' solutions near their parabolic limits. J. Math. Phys., 8(8):4334-4356, 1994.

[13] K.R. Oldham and J. Spanier. The Fractional Calculus. Academic Press, New York, 1974.

[14] Jeffrey O. Powell. Two coefficient reconstruction via a trace formulae in a one-dimensional inverse scattering problem. Inverse Problems, 11(1):275, 1995.

[15] A. P. Prudnikov, Yu. A. Brychkov, and O. I. L. Marichev. Integrals and Series. Gordon \& Breach Science Publishers, New York, 1986.

[16] Michael E. Taylor. Pseudodifferential Operators. Princeton Mathematical Series No. 34. Princeton University Press, Princeton, New Jersey, 1981. 
[17] C. R. Vogel. Wave Splitting for some Nonhyperbolic Time-Dependent PDEs, chapter 10, pages 129138. In Corones et al. [4], 1992.

[18] D. J. N. Wall. On the numerical solution of a functional differential equation pertaining to a wave equation, chapter 13, pages 169-186. In Corones et al. [4], 1992.

[19] V.H. Weston. Factorisation of the dissipative wave equation and inverse scattering. J. Math. Phys, $29: 2205-2218,1988$.

\section{Appendix A. Derivation of Equation (3)}

We provide a note on the derivation of the second order functional differential equation (3). Integration of Cattaneo's equation (1) shows it can be written as

$$
q=-\frac{k}{\tau} \mathrm{J} \partial_{x} T
$$

and then conservation equation (2) can be rewritten as

$$
c_{v} \rho \partial_{t} T-\partial_{x}\left(\frac{k}{\tau} \mathrm{J} \partial_{x} T\right)+q_{\ell}=r .
$$

Now to get a second order equation it is necessary to differentiate this equation with respect to $t$, so yielding on also multiplying both sides by $\tau / k$

$$
\kappa^{-2} \tau \partial_{t}^{2} T+\frac{\tau}{k} \partial_{t} q_{\ell}-\frac{\tau}{k} \partial_{x}\left(\frac{k}{\tau} \partial_{x} T\right)+\frac{\tau}{k} \partial_{x}\left(\tau^{-1} \frac{k}{\tau} \rrbracket \partial_{x} T\right)=\frac{\tau}{k} \partial_{t} r
$$

In this equation use has been made of the property of the exponential function being its own derivative. Some manipulation then enables the last term on the left-hand-side of this equation to be rewritten as

$$
\frac{\tau}{k} \partial_{x}\left(\tau^{-1} \frac{k}{\tau} \mathrm{J} \partial_{x} T\right)=\left[\left(\partial_{x} \tau^{-1}\right) J \partial_{x} T+\kappa^{-2} \partial_{t} T+\frac{1}{k} q_{\ell}-\frac{1}{k} r\right],
$$

and then (3) is found.

\section{ApPendix B. Asymptotic Forms of The Operators}

We list here the asymptotic forms of the various kernels in the text. On noting

$$
\begin{aligned}
& \mathrm{I}_{n}(a x) \approx\left(\frac{a x}{2}\right)^{n}\left(1+\mathcal{O}\left(x^{2}\right)\right) \text { for } x<<1, \\
& \mathrm{I}_{n}(a x) \approx \frac{e^{a x}}{\sqrt{2 \pi a x}}\left(1+\mathcal{O}\left(x^{-1}\right)\right) \text { for } x>>1,
\end{aligned}
$$

the following can be found

$$
\begin{aligned}
\lim _{\tau \rightarrow 0} \kappa K(t) & =\lim _{\tau \rightarrow 0} \frac{\kappa}{\sqrt{\tau}} e^{-t /(2 \tau)} \mathrm{I}_{0}(t /(2 \tau)) \\
& =\kappa h(t)(1+\mathcal{O}(\tau)) \\
\lim _{\tau \rightarrow 0} \kappa^{-1} \mathbb{K}^{-1} f(t) & =\lim _{\tau \rightarrow 0} \kappa^{-1} \partial_{t} \mathbb{K} \circ\left(\tau \partial_{t}+1\right) f(t) \\
& =\kappa^{-1} \partial_{t} \mathbb{H} \circ(1+\mathcal{O}(\tau)) f(t)
\end{aligned}
$$




$$
\begin{aligned}
\lim _{\tau \rightarrow \infty} \kappa K(t) & =\lim _{\tau \rightarrow \infty} \frac{\kappa}{\sqrt{\tau}} e^{-t /(2 \tau)} \mathrm{I}_{0}(t /(2 \tau)) \\
& =c\left(1+\mathcal{O}\left(\tau^{-2}\right)\right) \\
\lim _{\tau \rightarrow \infty} \kappa^{-1} K^{-1} f(t) & =\lim _{\tau \rightarrow \infty} \kappa^{-1} \sqrt{\tau}\left(\partial_{t}+\frac{1}{2 \tau}(1+\mathbb{L})\right) f(t) \\
& =c^{-1} \partial_{t} f(t) \\
& \lim _{\tau \rightarrow \infty} L(t) / \sqrt{\tau}=0
\end{aligned}
$$

Department of Mathematics \& Statistics, University of Canterbury, Christchurch, 1 , New Zealand

E-mail address: D. Wallomath. canterbury.ac.nz

Division of Mechanics, Chalmers University of Technology, S-412 96 Gothenburg, SweDEN

E-mail address: peolomec.chalmers.se 